\title{
QUEEN'S
UNIVERSITY
BELFAST
}

\section{New method based on the UNIFAC-VISCO model for the estimation of dynamic viscosity of (ionic liquid + molecular solvent) binary} mixtures

Zhao, N., \& Jacquemin, J. (2017). New method based on the UNIFAC-VISCO model for the estimation of dynamic viscosity of (ionic liquid + molecular solvent) binary mixtures. Fluid Phase Equilibria, 449, 41-51. https://doi.org/10.1016/j.fluid.2017.06.006

Published in:

Fluid Phase Equilibria

Document Version:

Peer reviewed version

Queen's University Belfast - Research Portal:

Link to publication record in Queen's University Belfast Research Portal

\author{
Publisher rights \\ (C) 2017 Elsevier B.V. All rights reserved. \\ This manuscript version is made available under the CC-BY-NC-ND 4.0 license http://creativecommons.org/licenses/by-nc-nd/4.0/,which \\ permits distribution and reproduction for noncommercial purposes, provided the author and source are cited.
}

\section{General rights}

Copyright for the publications made accessible via the Queen's University Belfast Research Portal is retained by the author(s) and / or other copyright owners and it is a condition of accessing these publications that users recognise and abide by the legal requirements associated with these rights.

\section{Take down policy}

The Research Portal is Queen's institutional repository that provides access to Queen's research output. Every effort has been made to ensure that content in the Research Portal does not infringe any person's rights, or applicable UK laws. If you discover content in the

Research Portal that you believe breaches copyright or violates any law, please contact openaccess@qub.ac.uk. 


\section{New Method Based on the UNIFAC-VISCO Model for the \\ Estimation of Dynamic Viscosity of (Ionic Liquid + Molecular Solvent) \\ Binary Mixtures}

$$
\text { Nan Zhao }{ }^{1} \text {, Johan Jacquemin }{ }^{1,2, *}
$$

${ }^{1}$ School of Chemistry and Chemical Engineering, Queen's University Belfast, Belfast, BT9 5AG, U.K.

2 Université Francois Rabelais, Laboratoire PCM2E, Parc de Grandmont 37200 Tours, France

Corresponding Author: *E-mail: ji@univ-tours.fr or johan.jacquemin@qub.ac.uk 


\section{ABSTRACT}

The modified UNIFAC-VISCO model was applied to evaluate the viscosity of (ionic liquid + molecular solvent) mixtures as the function of the composition and temperature at atmospheric pressure. The values of interaction parameters between ionic groups, $\alpha_{\text {ion/ion }}$ were collected from our previous paper (Zhao et al. J. Chem. Eng. Data 61 (2016) 3908-3921), while the parameters between the common organic groups, $\alpha_{\text {organic/organic }}$ were taken from Chevalier et al. (Chem. Eng. Sci. 49 (1994) 1799-1806). Another 376 unknown interaction parameters ( $\alpha_{\text {organic/organic }}$ and $\alpha_{\text {ion/organic }}$ ) were calculated by regression of 3365 experimental binary viscosity data for 119 different binary systems as a function of temperature and component composition. Then, this model was assessed through the evaluation of 781 viscosity data points for 24 different binary systems not originally included in the correlation set. The relative absolute average deviation (RAAD\%) of the correlation and evaluation for the viscosity of the investigated binary mixtures is close to $5.0 \%$ and $7.7 \%$, respectively, which proves that the UNIFAC-VISCO based method proposed in this work is reliable for the estimation of the viscosity of ionic liquid-based binary mixtures.

Keywords: UNIFAC-VISCO; Ionic Liquids; Molecular Solvents; Viscosity; Group Contribution Model 


\section{INTRODUCTION}

In recent years, ionic liquids (ILS) have attracted the attention of scientific communities due to their unique properties, such as chemical and thermal stability, high ionic conductivity, low vapor pressure, and easy tuning of their physicochemical properties by altering the cation, anion, or substituent [1]. Among the various properties, viscosity property is of considerable importance in the design of heat transfer equipment, liquid-liquid extractors, process piping, and other units [2]. For industrial applications, IL-cosolvent mixtures are promising alternatives to traditional organic liquids, since pure IL mixing with the organic liquid can confer a suitable viscosity to the mixture [1]. Consequently, many modeling efforts have been reported in the literature [1-13] regarding the viscosity of IL-cosolvent mixtures, used as electrolyte solutions.

In this regard, literature models can be categorized as (i) simple correlation equations, such as the Seddon [3], Grunberg-Nissan [9], and McAllister models [10]; (ii) Eyring's theory based methods, like the Eyring-UNIQUAC [11], Eyring-NRTL [12], Eyring-MTSM [8], Eyring-Flory-Huggins [7], and Eyring-Wilson models [13]; and (iii) predictive methods, for example, UNIFAC-VISCO [6] and ASOG-VISCO models [6].

Wang et al. [2] compared the Eyring-UNIQUAC model and the Eyring-NRTL model ( $\alpha$ fixed at 0.25 ) in the case of 35 binary mixtures containing ILs with a total of 1014 data points. The authors highlighted that a better evaluation of the viscosity could be achieved by using the Eyring-UNIQUAC (RAAD\% of 2.6\%) than by using Eyring-NRTL 
(RAAD\% of 3.5\%). Bajić et al. [6] used the Seddon, Grunberg-Nissan, McAlister, EyringUNIQUAC and Eyring-NRTL models to correlate the viscosity of 11 (IL + solvent) binary systems covering a wide temperature range. Within, McAlister and the threeparameter Eyring-NRTL models showed the best results with average percentage deviations $\left(P D_{\max }\right)$ below $1 \%$ in all cases. It should be noted that the reference in the denominator of the percentage deviation $\left(P D_{\max }\right)$ calculation is the maximum of experimental values. The Seddon model gives the highest deviation above $1 \%$ in all cases when compared with other correlative models, except for three cases; (2butanone $\left.+\left[\mathrm{C}_{4} \mathrm{mim}\right]\left[\mathrm{BF}_{4}\right]\right),\left(\mathrm{H}_{2} \mathrm{O}+\left[\mathrm{C}_{3} \mathrm{mmim}\right]\left[\mathrm{BF}_{4}\right]\right)$, and (methyl methacrylate + [ $\left.\left.\mathrm{C}_{4} \mathrm{mim}\right]\left[\mathrm{PF}_{6}\right]\right)$. Fang et al. [7] proposed a method based on the Eyring's absolute reaction rate theory, which was coupled with the Flory-Huggins equation, to estimate the viscosity of 527 binary mixtures including 63 (IL + solvent) mixtures. The RAAD\% of the proposed method assessed using 2554 data points of the 63 ionic liquid containing systems is close to $3.73 \%$ which shows a better viscosity predictive capability than the Grunberg-Nissan equation (7.42\%), for example. Furthermore, Ciocirlan et al. [1] tested 6 methods (i.e. G-N, McAllister, Eyring-Flory-Huggins, EyringUNIQUAC, Eyring-NRTL, and Eyring-Wilson) in terms of four binary systems $\left(\left[\mathrm{C}_{2} \mathrm{mim}\right]\left[\mathrm{BF}_{4}\right]+\right.$ dimethyl sulfoxide / ethylene glycol / acetonitrile / 1,4-dioxane $)$ at (293.15, 298.15, 303.15, 313.15, 323.15, 333.15, 343.15, and 353.15) K. The McAllister model gives the best viscosity evaluation below $1.34 \%$ quantified by RAAD\% at each temperature for three systems ([C $\left.\mathrm{C}_{2} \mathrm{mim}\right]\left[\mathrm{BF}_{4}\right]+$ dimethyl sulfoxide / acetonitrile / 1,4- 
dioxane). For the other system, $\left[\mathrm{C}_{2} \mathrm{mim}\right]\left[\mathrm{BF}_{4}\right]+$ ethylene glycol, the best result was reported using the Eyring-Flory-Huggins based method with a RAAD\% of $1.54 \%$, while the McAllister method also showed a good result with RAAD\% of $1.60 \%$. The poorest result estimated for these four systems is with the G-N model, ranging from $1.93 \%$ up to $6.01 \%$ calculated by RAAD\% at the eight temperatures. For the series of Eyringbased models, Eyring-Wilson gives the poorest results with a RAAD\% of $2.1 \%$ and $2.9 \%$ in two systems containing dimethyl sulfoxide, and acetonitrile, respectively. EyringFlory-Huggins shows the poorest estimation with a $1.5 \%$ RAAD\% in the system with ethylene glycol and also a poor result with $1.1 \%$ of RAAD\% in the 1,4-dioxane containing system. Generally, the other two Eyring-based models (i.e. UNIQUAC and NRTL) show a better evaluation, the RAAD\% are lower than $1.9 \%$ at each temperature point for all the four investigated systems. Atashrouz et al. [8] reported an Eyring's theory method coupled with a modified two-suffix-margules model (Eyring-MTSM). To validate the capability of the Eyring-MTSM model, the authors also made a clear comparison with the Eyring-NRTL model using 5512 experimental data points for 122 IL-based mixtures. Based on this work, these authors demonstrated the higher accuracy of the Eyring-MTSM method, with a RAAD\% close to $2 \%$ compared to $2.8 \%$ observed using the Eyring-NRTL model.

Generally, correlative models often yield lower deviations, but require enough experimental data for the determination of parameters in the correlation equations. However, a significant advantage of the predictive models is that a given property, such 
as viscosity could be evaluated for unknown mixtures by using a set of interaction parameters regressed using well-defined training datasets. Two predictive methods, based on the UNIFAC-VISCO and ASOG-VISCO models, respectively were applied for modeling the viscosity of IL-based mixtures [6]. Bajić et al. [6] calculated the interaction parameters $\left(\alpha_{m n}\right)$ between the group $\left(\left[\mathrm{C}_{2} \mathrm{mim}\right]\left[\mathrm{C}_{2} \mathrm{SO}_{4}\right],[-\mathrm{mim}]\left[\mathrm{BF}_{4}\right],[-\right.$ $\operatorname{mim}]\left[\mathrm{PF}_{6}\right]$, and $\left.\left[\mathrm{C}_{4} \mathrm{mim}\right]\left[\mathrm{NO}_{3}\right]\right)$ and the organic functional groups by correlating experimental data of 11 binary mixtures. However, the determination of the groups in the ionic liquid components is not clear and uniform, sometimes on the basis of the whole IL (such as $\left[\mathrm{C}_{2} \mathrm{mim}\right]\left[\mathrm{C}_{2} \mathrm{SO}_{4}\right]$ and $\left[\mathrm{C}_{4} \mathrm{mim}\right]\left[\mathrm{NO}_{3}\right]$ ) and sometimes on the basis of the core structure of the IL $\left([-\mathrm{mim}]\left[\mathrm{BF}_{4}\right]\right.$ and $\left.[-\mathrm{mim}]\left[\mathrm{PF}_{6}\right]\right)$. In fact more work is requested to assess properly the quality of the proposed method using novel and original viscosity datasets.

In this work, we aim to develop the UNIFAC-VISCO model for correlation, and then evaluation of the viscosity of (IL + molecular solvent) binary mixtures. The method used for molecules' cleavage is different from the division method reported by Bajić et al. [6]. Each cation or anion, constituting the IL, is defined as an individual group, which is coincident with the method of ionic groups determination for the viscosity estimation of pure ILs and binary ILs mixtures reported in our previous work $[14,15]$. As a result, 119 binary systems with a total of 3365 experimental data points were regressed altogether to obtain the interaction parameters between functional groups ( $\alpha_{\text {ion/organic }}$ and $\left.\alpha_{\text {organic/organic }}\right)$. Then other 24 binary mixtures, not involved in 
the training set, were compared from the pure evaluated values to test the validity of the interaction parameters and thus the model developed in the current study. 


\section{DEVELOPMENT OF THE PROPOSED MODEL}

2.1 Database. In this work, 4146 experimental viscosity data at $0.1 \mathrm{MPa}$ for 143 binary mixtures were collected from the NIST database, as a function of the composition over a broad temperature range (from $273.15 \mathrm{~K}$ to $363.15 \mathrm{~K}$ ). As discrepancies between the literature datasets for a given system could be observed, the experimental viscosities for each binary system, used to establish the UNIFAC-VISCO model, were taken only from one reference. The UNIFAC-VISCO model calculates the binary viscosities on the basis of the pure components' properties. The references that also report the pure components' densities and viscosities were preferred, thus identical measurement uncertainties for the properties of pure ILS and mixtures containing ILs increase the accuracy of the optimized binary interaction parameters. If the values of the pure component's properties are not provided in the selected reference, the molar volume and viscosity of pure ILs were calculated by using the methods previously proposed by our group [14-17]. In the database, 3365 data points of 119 binary mixtures from literature [18-86], as a function of temperature and component composition, were used for optimization of interaction parameters and 781 data points of another 24 binary mixtures were used to validate the performance of the proposed model. Table 1 shows the list of the selected binary systems along with their corresponding temperature range and composition. 
Table 1. List of the selected (ionic liquid + molecular solvent) binary mixtures

\begin{tabular}{|c|c|c|c|c|c|c|c|c|c|}
\hline No. & Ionic Component (1) & Organic Component (2) & $\begin{array}{c}\text { Temperature } \\
\text { Range (K) }\end{array}$ & Range of $x_{1}(\mathrm{~mol} \%)$ & $\begin{array}{c}\text { No. of Data } \\
\text { Points }\end{array}$ & $\begin{array}{l}\text { Measurement } \\
\text { Uncertainty }\end{array}$ & $\begin{array}{c}\text { RAAD } \\
(\%)\end{array}$ & Ref. $(\mu)$ & Ref. $\left(\rho_{I L}\right)$ \\
\hline \multicolumn{10}{|c|}{ Correlation Set } \\
\hline 1 & {$\left[\mathrm{C}_{2} \mathrm{mim}\right]\left[\mathrm{C}_{2} \mathrm{SO}_{4}\right]$} & 1-propanol & 298.15 to 328.15 & 0.0273 to 0.8381 & 27 & $\pm 0.006(\mathrm{mPa} \cdot \mathrm{s})$ & 3.62 & [18] & [18] \\
\hline 2 & {$\left[\mathrm{C}_{2} \mathrm{mim}\right]\left[\mathrm{C}_{2} \mathrm{SO}_{4}\right]$} & methanol & 298.15 to 328.15 & 0.0083 to 0.8654 & 33 & $\pm 0.006(\mathrm{mPa} \cdot \mathrm{s})$ & 6.35 & [18] & [18] \\
\hline 3 & {$\left[\mathrm{C}_{2} \mathrm{mim}\right]\left[\mathrm{C}_{2} \mathrm{SO}_{4}\right]$} & ethanol & 298.15 to 328.15 & 0.0137 to 0.9377 & 30 & $\pm 0.006(\mathrm{mPa} \cdot \mathrm{s})$ & 4.34 & [19] & [19] \\
\hline 4 & {$\left[\mathrm{C}_{2} \mathrm{mim}\right]\left[\mathrm{BF}_{4}\right]$} & methanol & 278.15 to 318.15 & 0.0499 to 0.9476 & 65 & $2 \%$ & 2.73 & [20] & [20] \\
\hline 5 & {$\left[\mathrm{C}_{2} \mathrm{mim}\right]\left[\mathrm{BF}_{4}\right]$} & ethanol & 288.15 to 318.15 & 0.5447 to 0.9054 & 24 & $0.3 \%$ & 3.57 & [21] & [20] \\
\hline 6 & {$\left[\mathrm{C}_{2} \mathrm{mim}\right]\left[\mathrm{C}_{2} \mathrm{SO}_{4}\right]$} & water & 298.15 to 328.15 & 0.0494 to 0.9285 & 24 & $\pm 0.006(\mathrm{mPa} \cdot \mathrm{s})$ & 2.54 & [19] & [19] \\
\hline 7 & {$\left[\mathrm{C}_{2} \mathrm{mim}\right]\left[\mathrm{BF}_{4}\right]$} & dimethyl sulfoxide & 303.15 to 333.15 & 0.0998 to 0.8999 & 36 & $<0.6 \%$ & 2.04 & [22] & [22] \\
\hline 8 & {$\left[\mathrm{C}_{2} \mathrm{mim}\right]\left[\mathrm{BF}_{4}\right]$} & $\mathrm{N}, \mathrm{N}$-dimethylformamide & 303.15 to 333.15 & 0.1001 to 0.8984 & 36 & $<0.6 \%$ & 0.97 & [22] & [22] \\
\hline 9 & {$\left[\mathrm{C}_{2} \mathrm{mim}\right]\left[\mathrm{BF}_{4}\right]$} & dimethylacetamide & 303.15 to 333.15 & 0.1006 to 0.9004 & 36 & $<0.6 \%$ & 1.47 & [22] & [22] \\
\hline 10 & {$\left[\mathrm{C}_{4} \mathrm{mim}\right]\left[\mathrm{BF}_{4}\right]$} & water & 303.15 to 353.15 & 0.1001 to 0.8998 & 72 & $\pm 0.01(\mathrm{mPa} \cdot \mathrm{s})$ & 7.41 & [23] & [23] \\
\hline 11 & {$\left[\mathrm{C}_{4} \mathrm{mim}\right]\left[\mathrm{BF}_{4}\right]$} & methanol & 303.15 to 333.15 & 0.0999 to 0.8997 & 36 & $\pm 0.35 \%$ & 3.50 & [24] & [24] \\
\hline 12 & {$\left[\mathrm{C}_{4} \mathrm{mim}\right]\left[\mathrm{BF}_{4}\right]$} & dimethyl sulfoxide & 293.15 to 353.15 & 0.0722 to 0.9155 & 63 & $\pm 0.1 \%$ & 3.11 & [25] & [25] \\
\hline 13 & {$\left[\mathrm{C}_{4} \mathrm{mim}\right]\left[\mathrm{BF}_{4}\right]$} & 2-butanone & 298.15 & 0.0498 to 0.8995 & 13 & $\pm 0.3 \%$ & 2.36 & [26] & [26] \\
\hline 14 & {$\left[\mathrm{C}_{4} \mathrm{mim}\right]\left[\mathrm{BF}_{4}\right]$} & dimethylacetamide & 303.15 to 333.15 & 0.1001 to 0.8994 & 36 & $\pm 0.35 \%$ & 2.56 & [24] & [24] \\
\hline 15 & {$\left[\mathrm{C}_{4} \mathrm{mim}\right]\left[\mathrm{BF}_{4}\right]$} & $\mathrm{N}, \mathrm{N}$-dimethylformamide & 298.15 & 0.053 to 0.8976 & 13 & $\pm 0.3 \%$ & 2.46 & [26] & [26] \\
\hline 16 & {$\left[\mathrm{C}_{4} \mathrm{mim}\right]\left[\mathrm{BF}_{4}\right]$} & acetonitrile & 298.15 & 0.0487 to 0.9018 & 13 & $\pm 0.3 \%$ & 4.73 & [26] & [26] \\
\hline 17 & {$\left[\mathrm{C}_{4} \mathrm{mim}\right]\left[\mathrm{BF}_{4}\right]$} & ethyl formate & 298.15 & 0.05 to 0.8996 & 13 & $\pm 0.3 \%$ & 4.56 & [27] & [27] \\
\hline 18 & {$\left[\mathrm{C}_{4} \mathrm{mim}\right]\left[\mathrm{BF}_{4}\right]$} & triethanolamine & 303.15 to 343.15 & 0.1301 to 0.7054 & 25 & $<0.1 \%$ & 11.61 & [28] & [28] \\
\hline
\end{tabular}




\begin{tabular}{|c|c|c|c|c|c|c|c|c|c|}
\hline 19 & {$\left[\mathrm{C}_{4} \mathrm{mim}\right]\left[\mathrm{BF}_{4}\right]$} & methyl acetate & 298.15 & 0.0501 to 0.8999 & 13 & $\pm 0.3 \%$ & 4.58 & [27] & [27] \\
\hline 20 & {$\left[\mathrm{C}_{4} \mathrm{mim}\right]\left[\mathrm{BF}_{4}\right]$} & pyridine & 298.15 & 0.193 to 0.8941 & 8 & $\pm 1 \%$ & 0.28 & [29] & [29] \\
\hline 21 & {$\left[\mathrm{C}_{4} \mathrm{mim}\right]\left[\mathrm{BF}_{4}\right]$} & chloroform & 298.15 & 0.1509 to 0.8921 & 9 & $\pm 1 \%$ & 1.52 & [29] & [29] \\
\hline 22 & {$\left[\mathrm{C}_{4} \mathrm{mim}\right]\left[\mathrm{BF}_{4}\right]$} & dichloromethane & 298.15 & 0.1605 to 0.9 & 9 & $\pm 1 \%$ & 3.02 & [29] & [29] \\
\hline 23 & {$\left[\mathrm{C}_{4} \mathrm{mim}\right]\left[\mathrm{BF}_{4}\right]$} & $\mathrm{N}$-methyl-2-pyrrolidone & 298.15 to 318.15 & 0.3049 to 0.7945 & 15 & $-b$ & 1.59 & [30] & [30] \\
\hline 24 & {$\left[\mathrm{C}_{2} \mathrm{mim}\right]\left[\mathrm{NTf}_{2}\right]$} & ethanol & 278.15 to 338.15 & 0.051 to 0.907 & 40 & $0.1 \%$ & 1.96 & [31] & [31] \\
\hline 25 & {$\left[\mathrm{C}_{2} \mathrm{mim}\right]\left[\mathrm{NTf}_{2}\right]$} & $\mathrm{N}$-methyl-2-pyrrolidone & 293.15 to 323.15 & 0.0978 to 0.9002 & 63 & $<0.1 \%$ & 3.24 & [32] & [32] \\
\hline 26 & {$\left[\mathrm{C}_{2} \operatorname{mim}\right]\left[\mathrm{NTf} f_{2}\right]$} & benzene & 298.15 to 328.15 & 0.508 to 0.865 & 16 & $\pm 0.35 \%$ & 1.67 & [33] & [33] \\
\hline 27 & {$\left[\mathrm{C}_{4} \mathrm{mim}\right]\left[\mathrm{NTf}_{2}\right]$} & thiophene & 298.15 to 328.15 & 0.23 to 0.911 & 20 & $\pm 0.35 \%$ & 4.17 & [33] & [33] \\
\hline 28 & {$\left[\mathrm{C}_{4} \mathrm{mim}\right]\left[\mathrm{NTf}_{2}\right]$} & $\gamma$-butyrolactone & 293.15 to 323.15 & 0.1005 to 0.8995 & 63 & $1 \%$ & 3.06 & [34] & [34] \\
\hline 29 & {$\left[\mathrm{C}_{4} \mathrm{mim}\right]\left[\mathrm{NTf}_{2}\right]$} & 2,2,2-trifluoroethanol & 278.15 to 333.15 & 0.1252 to 0.9123 & 72 & $2 \%$ & 4.59 & [35] & [35] \\
\hline 30 & {$\left[\mathrm{C}_{4} \mathrm{mim}\right]\left[\mathrm{NTf}_{2}\right]$} & isopropyl acetate & 298.15 & 0.1082 to 0.9486 & 11 & $\pm 0.5 \%$ & 4.65 & [36] & [36] \\
\hline 31 & {$\left[\mathrm{C}_{6} \mathrm{mim}\right]\left[\mathrm{NTf}_{2}\right]$} & methyl diethanolamine & 303.15 to 323.15 & 0.101 to 0.9011 & 45 & $--^{b}$ & 6.97 & [37] & [37] \\
\hline 32 & {$\left[\mathrm{C}_{6} \mathrm{mim}\right]\left[\mathrm{NTf}_{2}\right]$} & 1-propanol & 278.15 to 338.15 & 0.048 to 0.8999 & 40 & $-c$ & 3.70 & [38] & [38] \\
\hline 33 & {$\left[\mathrm{C}_{6} \mathrm{mim}\right]\left[\mathrm{NTf}_{2}\right]$} & chloroform & 288.2 to 318.2 & 0.074 to 0.906 & 40 & $2 \sim 3 \%$ & 4.18 & [39] & $-a^{a}$ \\
\hline 34 & {$\left[\mathrm{C}_{6} \mathrm{mim}\right]\left[\mathrm{NTf}_{2}\right]$} & 1-octene & 283.15 to 348.15 & 0.72 to 0.95 & 16 & $\pm 1 \%$ & 3.07 & [40] & [41] \\
\hline 35 & {$\left[\mathrm{C}_{6} \mathrm{mim}\right]\left[\mathrm{BF}_{4}\right]$} & ethanol & 288.15 to 318.15 & 0.098 to 0.9 & 36 & $0.3 \%$ & 5.30 & [21] & [42] \\
\hline 36 & {$\left[\mathrm{C}_{6} \mathrm{mim}\right]\left[\mathrm{BF}_{4}\right]$} & 2-methyl-2-propanol & 298.15 & 0.1017 to 0.9 & 9 & $\pm 0.01(\mathrm{mPa} \cdot \mathrm{s})$ & 7.98 & [43] & [43] \\
\hline 37 & {$\left[\mathrm{C}_{6} \mathrm{mim}\right]\left[\mathrm{BF}_{4}\right]$} & 3-amino-1-propanol & 303.15 to 308.15 & 0.0536 to 0.9204 & 18 & $\pm 0.01(\mathrm{mPa} \cdot \mathrm{s})$ & 4.86 & [43] & [43] \\
\hline 38 & {$\left[\mathrm{C}_{6} \mathrm{mim}\right]\left[\mathrm{BF}_{4}\right]$} & water & 288.15 to 318.15 & 0.28 to 0.8899 & 32 & $\pm 0.35 \%$ & 3.79 & [44] & $--^{a}$ \\
\hline 39 & {$\left[\mathrm{C}_{6} \mathrm{mim}\right]\left[\mathrm{BF}_{4}\right]$} & propylamine & 298.15 & 0.0988 to 0.8475 & 9 & $\pm 0.01(\mathrm{mPa} \bullet \mathrm{s})$ & 33.98 & [43] & [43] \\
\hline 40 & {$\left[\mathrm{C}_{6} \mathrm{mim}\right]\left[\mathrm{BF}_{4}\right]$} & 2-butanone & 298.15 & 0.0492 to 0.8931 & 13 & $\pm 1 \%$ & 1.83 & [45] & [45] \\
\hline 41 & {$\left[\mathrm{C}_{6} \mathrm{mim}\right]\left[\mathrm{BF}_{4}\right]$} & ethyl acetate & 298.15 & 0.0498 to 0.8908 & 13 & $\pm 1 \%$ & 0.88 & [45] & [45] \\
\hline 42 & {$\left[\mathrm{C}_{6} \mathrm{mim}\right]\left[\mathrm{BF}_{4}\right]$} & tetrahydrofuran & 298.15 & 0.0498 to 0.8925 & 13 & $\pm 1 \%$ & 1.45 & [45] & [45] \\
\hline
\end{tabular}




\begin{tabular}{|c|c|c|c|c|c|c|c|c|c|}
\hline 43 & {$\left[\mathrm{C}_{4} \mathrm{py}\right]\left[\mathrm{NTf}_{2}\right]$} & water & 298.15 to 363.15 & 0.752 & 14 & $\pm 0.35 \%$ & 2.46 & [46] & [46] \\
\hline 44 & {$\left[\mathrm{C}_{4} \mathrm{py}\right]\left[\mathrm{BF}_{4}\right]$} & dichloromethane & 298.15 & 0.08071 to 0.94508 & 8 & $1 \%$ & 7.72 & [47] & $-a^{a}$ \\
\hline 45 & {$\left[\mathrm{C}_{4} \mathrm{py}\right]\left[\mathrm{BF}_{4}\right]$} & water & 298.15 & 0.024 to 0.82817 & 9 & $1 \%$ & 4.43 & [47] & $-a^{a}$ \\
\hline 46 & {$\left[\mathrm{C}_{4} \mathrm{py}\right]\left[\mathrm{BF}_{4}\right]$} & methanol & 298.15 & 0.05255 to 0.91576 & 9 & $1 \%$ & 3.87 & [47] & $-\mathrm{a}^{\mathrm{a}}$ \\
\hline 47 & {$\left[\mathrm{C}_{8} \mathrm{mim}\right]\left[\mathrm{NTf}_{2}\right]$} & 2-propanol & 298.15 & 0.1034 to 0.9669 & 12 & $\pm 0.5 \%$ & 1.76 & [48] & [48] \\
\hline 48 & {$\left[\mathrm{C}_{8} \mathrm{mim}\right]\left[\mathrm{NTf}_{2}\right]$} & isopropyl acetate & 298.15 & 0.1009 to 0.9585 & 11 & $\pm 0.5 \%$ & 1.20 & [48] & [48] \\
\hline 49 & {$\left[\mathrm{C}_{8} \mathrm{mim}\right]\left[\mathrm{NTf}_{2}\right]$} & methanol & 298.15 & 0.098 to 0.9346 & 10 & $\pm 0.5 \%$ & 3.09 & [49] & [49] \\
\hline 50 & {$\left[\mathrm{C}_{8} \mathrm{mim}\right]\left[\mathrm{BF}_{4}\right]$} & 1-propanol & 298.15 & 0.0473 to 0.9118 & 13 & $-{ }^{b}$ & 7.26 & [50] & [50] \\
\hline 51 & {$\left[\mathrm{C}_{8} \mathrm{mim}\right]\left[\mathrm{BF}_{4}\right]$} & 2-butanone & 298.15 & 0.0482 to 0.899 & 13 & $\pm 0.01(\mathrm{mPa} \cdot \mathrm{s})$ & 1.64 & [51] & [51] \\
\hline 52 & {$\left[\mathrm{C}_{8} \mathrm{mim}\right]\left[\mathrm{BF}_{4}\right]$} & methanol & 298.15 & 0.0495 to 0.9049 & 13 & $-b$ & 1.79 & [50] & [50] \\
\hline 53 & {$\left[\mathrm{C}_{8} \mathrm{mim}\right]\left[\mathrm{BF}_{4}\right]$} & ethyl acetate & 298.15 & 0.047 to 0.8908 & 13 & $\pm 0.01(\mathrm{mPa} \cdot \mathrm{s})$ & 3.88 & [51] & [51] \\
\hline 54 & {$\left[\mathrm{C}_{4} \mathrm{mim}\right]\left[\mathrm{PF}_{6}\right]$} & 2-butanone & 298.15 & 0.0513 to 0.9005 & 13 & $\pm 0.2 \%$ & 5.81 & [52] & [52] \\
\hline 55 & {$\left[\mathrm{C}_{4} \mathrm{mim}\right]\left[\mathrm{PF}_{6}\right]$} & acetonitrile & 298.15 & 0.1082 to 0.901 & 10 & $\pm 1 \%$ & 1.87 & [29] & [29] \\
\hline 56 & {$\left[\mathrm{C}_{4} \mathrm{mim}\right]\left[\mathrm{PF}_{6}\right]$} & dichloromethane & 298.15 & 0.1322 to 0.9247 & 10 & $\pm 1 \%$ & 1.99 & [29] & [29] \\
\hline 57 & {$\left[\mathrm{C}_{4} \mathrm{mim}\right]\left[\mathrm{PF}_{6}\right]$} & chloroform & 298.15 & 0.2674 to 0.9143 & 8 & $\pm 1 \%$ & 2.84 & [29] & [29] \\
\hline 58 & {$\left[\mathrm{C}_{4} \mathrm{mim}\right]\left[\mathrm{PF}_{6}\right]$} & cyclopentanone & 298.15 & 0.0482 to 0.9064 & 13 & $\pm 0.2 \%$ & 1.19 & [52] & [52] \\
\hline 59 & {$\left[\mathrm{C}_{4} \mathrm{mim}\right]\left[\mathrm{PF}_{6}\right]$} & $\mathrm{N}, \mathrm{N}$-dimethylethanolamine & 288.15 to 323.15 & 0.0872 to 0.8779 & 72 & $\pm 0.1 \%$ & 5.01 & [53] & [53] \\
\hline 60 & {$\left[\mathrm{C}_{4} \mathrm{mim}\right]\left[\mathrm{PF}_{6}\right]$} & $\mathrm{N}, \mathrm{N}$-dimethylformamide & 303.15 to 323.15 & 0.0276 to 0.0988 & 9 & $0.35 \%$ & 2.08 & [54] & [54] \\
\hline 61 & {$\left[\mathrm{C}_{4} \mathrm{mim}\right]\left[\mathrm{PF}_{6}\right]$} & dimethyl sulfoxide & 298.15 & 0.05 to 0.893 & 13 & $\pm 0.5 \%$ & 2.28 & [55] & [55] \\
\hline 62 & {$\left[\mathrm{C}_{4} \mathrm{mim}\right]\left[\mathrm{PF}_{6}\right]$} & water & 298.15 & 0.7854 to 0.9482 & 5 & $\pm 1 \%$ & 2.13 & [29] & [29] \\
\hline 63 & {$\left[\mathrm{C}_{4} \mathrm{mim}\right]\left[\mathrm{PF}_{6}\right]$} & methanol & 298.15 & 0.0503 to 0.8889 & 13 & $\pm 0.5 \%$ & 6.42 & {$[55]$} & [55] \\
\hline 64 & {$\left[\mathrm{C}_{4} \mathrm{mim}\right]\left[\mathrm{PF}_{6}\right]$} & methyl methacrylate & 283.15 to 353.15 & 0.0993 to 0.9014 & 117 & $<2.612 \%$ & 4.72 & [56] & [56] \\
\hline 65 & {$\left[\mathrm{C}_{4} \mathrm{mim}\right]\left[\mathrm{PF}_{6}\right]$} & monoethanolamine & 288.15 to 323.15 & 0.0972 to 0.8815 & 72 & $\pm 0.1 \%$ & 2.43 & [53] & [53] \\
\hline 66 & {$\left[\mathrm{C}_{4} \mathrm{mim}\right]\left[\mathrm{PF}_{6}\right]$} & tetrahydrofuran & 298.15 & 0.0502 to 0.9595 & 13 & $\pm 0.5 \%$ & 2.73 & [55] & [55] \\
\hline
\end{tabular}




\begin{tabular}{|c|c|c|c|c|c|c|c|c|c|}
\hline 67 & {$\left[\mathrm{C}_{4} \mathrm{mim}\right]\left[\mathrm{PF}_{6}\right]$} & 2,2,2-trifluoroethanol & 278.15 to 333.15 & 0.0583 to 0.5368 & 60 & $2 \%$ & 11.03 & [35] & [35] \\
\hline 68 & {$\left[\mathrm{C}_{4} \mathrm{mim}\right][\mathrm{DCA}]$} & water & 278.15 to 358.15 & 0.5169 to 0.941 & 54 & $\pm 0.35 \%$ & 6.88 & [57] & [57] \\
\hline 69 & {$\left[\mathrm{C}_{4} \mathrm{mim}\right][\mathrm{DCA}]$} & $\gamma$-butyrolactone & 273.15 to 323.15 & 0.0504 to 0.8871 & 56 & $<0.5 \%$ & 2.17 & [58] & [58] \\
\hline 70 & {$\left[\mathrm{C}_{2} \mathrm{mim}\right][\mathrm{DCA}]$} & ethanol & 298.15 to 343.15 & 0.0673 to 0.898 & 40 & $0.65 \%$ & 4.01 & [59] & [59] \\
\hline 71 & {$\left[\mathrm{C}_{4} \mathrm{mpyrro}\right]\left[\mathrm{NTf}_{2}\right]$} & acetonitrile & 288.15 to 308.15 & 0.1924 to 0.8159 & 12 & $\pm 0.2 \%$ & 2.32 & {$[60]$} & [60] \\
\hline 72 & {$\left[\mathrm{C}_{4} \mathrm{mpyrro}\right]\left[\mathrm{NTf}_{2}\right]$} & $\mathrm{N}, \mathrm{N}$-dimethylformamide & 295.2 & 0.26 to 0.87 & 7 & $\pm 1 \%$ & 7.00 & [61] & $-a^{a}$ \\
\hline 73 & {$\left[\mathrm{C}_{4}\right.$ mpyrro] $\left[\mathrm{NTf}_{2}\right]$} & $\gamma$-butyrolactone & 298.15 to 323.15 & 0.1 to 0.8925 & 54 & $1 \%$ & 2.07 & [62] & [62] \\
\hline 74 & {$\left[\mathrm{C}_{4} \mathrm{mpyrro}\right]\left[\mathrm{NTf}_{2}\right]$} & methanol & 288.15 to 308.15 & 0.2054 to 0.7067 & 12 & $\pm 0.2 \%$ & 1.86 & {$[60]$} & [60] \\
\hline 75 & {$\left[\mathrm{C}_{4} \mathrm{mpyrro}\right]\left[\mathrm{NTf}_{2}\right]$} & propylene carbonate & 293.15 to 318.15 & 0.05 to 0.95 & 108 & $0.01(\mathrm{mPa} \cdot \mathrm{s})$ & 3.20 & [63] & [63] \\
\hline 76 & {$\left[\mathrm{C}_{4} \mathrm{mpyrro}\right][\mathrm{SCN}]$} & water & 298.15 to 348.15 & 0.0343 to 0.9147 & 48 & $\pm 0.1 \%$ & 7.50 & [64] & [64] \\
\hline 77 & {$\left[\mathrm{C}_{4} \mathrm{mim}\right][\mathrm{SCN}]$} & ethanol & 298.15 to 348.15 & 0.0473 to 0.9174 & 54 & $<1 \%$ & 8.38 & [65] & [65] \\
\hline 78 & {$\left[\mathrm{C}_{4} \mathrm{mim}\right][\mathrm{SCN}]$} & 1-hexanol & 298.15 to 348.15 & 0.0898 to 0.9576 & 60 & $<0.05 \%$ & 4.82 & [66] & [66] \\
\hline 79 & {$\left[\mathrm{C}_{4} \mathrm{mim}\right][\mathrm{SCN}]$} & 1-decanol & 298.15 to 348.15 & 0.0747 to 0.9587 & 60 & $<1 \%$ & 3.31 & [67] & [67] \\
\hline 80 & {$\left[\mathrm{C}_{4} \mathrm{mim}\right][\mathrm{SCN}]$} & methanol & 298.15 to 328.15 & 0.0154 to 0.9046 & 44 & $<1 \%$ & 3.42 & [65] & [65] \\
\hline 81 & {$\left[\mathrm{C}_{4} \mathrm{mim}\right][\mathrm{SCN}]$} & thiophene & 298.15 to 328.15 & 0.299 to 0.897 & 20 & $\pm 0.35 \%$ & 3.73 & [33] & [33] \\
\hline 82 & {$\left[\mathrm{C}_{4} \mathrm{mim}\right][\mathrm{SCN}]$} & benzene & 298.15 to 328.15 & 0.4906 to 0.7666 & 16 & $\pm 0.35 \%$ & 1.45 & [33] & [33] \\
\hline 83 & {$\left[\mathrm{C}_{1} \mathrm{mim}\right]\left[\mathrm{C}_{1} \mathrm{SO}_{4}\right]$} & water & 298.15 to 328.15 & 0.0515 to 0.9503 & 33 & $< \pm 0.2(\mathrm{mPa} \cdot \mathrm{s})$ & 5.72 & [68] & [68] \\
\hline 84 & {$\left[\mathrm{C}_{1} \mathrm{mim}\right]\left[\mathrm{C}_{1} \mathrm{SO}_{4}\right]$} & ethanol & 298.15 to 328.15 & 0.055 to 0.9331 & 33 & $< \pm 0.2(\mathrm{mPa} \cdot \mathrm{s})$ & 3.35 & [68] & [68] \\
\hline 85 & {$\left[\mathrm{C}_{4} \mathrm{mim}\right][\mathrm{OTf}]$} & benzene & 298.15 to 328.15 & 0.427 to 0.878 & 20 & $\pm 0.35 \%$ & 2.18 & [33] & [33] \\
\hline 86 & {$\left[\mathrm{C}_{2} \mathrm{mim}\right][\mathrm{OTf}]$} & water & 278.15 to 348.15 & 0.0648 to 0.7714 & 40 & $\pm 2 \%$ & 12.88 & [69] & [69] \\
\hline 87 & {$\left[\mathrm{C}_{2} \mathrm{mim}\right][\mathrm{OAc}]$} & ethanol & 298.15 to 343.15 & 0.1334 to 0.8941 & 40 & $0.65 \%$ & 6.57 & [59] & [59] \\
\hline 88 & {$\left[\mathrm{C}_{2} \mathrm{mim}\right][\mathrm{OAc}]$} & water & 298.15 to 343.15 & 0.0933 to 0.9008 & 40 & $0.65 \%$ & 8.96 & [59] & [59] \\
\hline 89 & {$\left[\mathrm{C}_{4} \mathrm{mim}\right][\mathrm{OAc}]$} & methyl diethanolamine & 293.15 to 343.15 & 0.2 to 0.8 & 24 & $\pm 0.5 \%$ & 9.52 & [70] & [70] \\
\hline 90 & {$\left[\mathrm{C}_{4} \mathrm{mim}\right][\mathrm{OAc}]$} & diethanolamine & 303.15 to 343.15 & 0.2 to 0.8 & 20 & $\pm 0.5 \%$ & 10.24 & [70] & [70] \\
\hline
\end{tabular}




\begin{tabular}{|c|c|c|c|c|c|c|c|c|c|}
\hline 91 & {$\left[\mathrm{C}_{4} \mathrm{mim}\right][\mathrm{OAc}]$} & acetonitrile & 298.15 & 0.1244 to 0.6794 & 4 & $\pm 2 \%$ & 7.59 & [71] & $-a^{a}$ \\
\hline 92 & {$\left[\mathrm{C}_{4} \mathrm{mim}\right][\mathrm{OAc}]$} & $\mathrm{N}, \mathrm{N}$-dimethylformamide & 298.15 & 0.1933 to 0.848 & 4 & $\pm 2 \%$ & 6.48 & [71] & $-a^{a}$ \\
\hline 93 & {$\left[\mathrm{P}_{66614}\right]\left[\mathrm{NTf}_{2}\right]$} & water & 298.15 to 358.15 & 0.912 & 13 & $\pm 0.35 \%$ & 4.91 & [72] & [72] \\
\hline 94 & {$\left[\mathrm{~N}_{1114}\right]\left[\mathrm{NTf} f_{2}\right]$} & 1,2-butanediol & 323.15 to 353.15 & 0.0969 to 0.7709 & 42 & $\pm 0.1 \%$ & 4.36 & [73] & [73] \\
\hline 95 & {$\left[\mathrm{~N}_{1114}\right][\mathrm{NTf} 2]$} & 2,3-butanediol & 323.15 to 343.15 & 0.1858 to 0.9121 & 30 & $\pm 0.1 \%$ & 5.69 & [73] & [73] \\
\hline 96 & {$\left[\mathrm{C}_{4} \mathrm{mmim}\right]\left[\mathrm{BF}_{4}\right]$} & dimethyl sulfoxide & 298.15 to 353.15 & 0.138 to 0.8785 & 56 & $\pm 0.1 \%$ & 2.40 & [74] & [74] \\
\hline 97 & {$\left[\mathrm{C}_{4} \mathrm{mmim}\right]\left[\mathrm{BF}_{4}\right]$} & acetonitrile & 298.15 to 343.15 & 0.1118 to 0.8423 & 42 & $\pm 0.1 \%$ & 4.58 & [74] & [74] \\
\hline 98 & {$\left[\mathrm{C}_{8} \operatorname{mim}\right] \mathrm{Cl}$} & methanol & 298.15 to 328.15 & 0.0197 to 0.9435 & 39 & $<1(\mathrm{mPa} \cdot \mathrm{s})$ & 9.98 & [75] & [75] \\
\hline 99 & {$\left[\mathrm{C}_{8} \mathrm{mim}\right] \mathrm{Cl}$} & ethanol & 298.15 to 328.15 & 0.0206 to 0.9551 & 27 & $<1(\mathrm{mPa} \cdot \mathrm{s})$ & 9.11 & [75] & [75] \\
\hline 100 & {$\left[\mathrm{C}_{2} \mathrm{mim}\right]\left[\mathrm{BF}_{4}\right]$} & water & 288.15 to 318.15 & 0.1009 to 0.9015 & 36 & $\pm 0.35 \%$ & 6.65 & [44] & $-a^{a}$ \\
\hline 101 & {$\left[\mathrm{C}_{4} \mathrm{mim}\right]\left[\mathrm{BF}_{4}\right]$} & ethanol & 288.15 to 318.15 & 0.0986 to 0.9235 & 36 & $0.3 \%$ & 10.65 & [21] & $-a^{a}$ \\
\hline 102 & {$\left[\mathrm{C}_{4} \mathrm{mim}\right]\left[\mathrm{BF}_{4}\right]$} & methyl formate & 298.15 & 0.05 to 0.8993 & 13 & $\pm 0.3 \%$ & 3.81 & [27] & [27] \\
\hline 103 & {$\left[\mathrm{C}_{4} \mathrm{mim}\right]\left[\mathrm{NTf}_{2}\right]$} & butyl acetate & 298.15 to 323.15 & 0.11 to 0.9056 & 54 & $3 \%$ & 12.69 & [76] & [76] \\
\hline 104 & {$\left[\mathrm{C}_{4} \mathrm{mim}\right]\left[\mathrm{NTf}_{2}\right]$} & ethanol & 298.15 & 0.1016 to 0.9207 & 12 & $\pm 0.5 \%$ & 2.24 & [77] & [77] \\
\hline 105 & {$\left[\mathrm{C}_{6} \mathrm{mim}\right]\left[\mathrm{BF}_{4}\right]$} & butylamine & 298.15 & 0.0492 to 0.8787 & 13 & $\pm 1 \%$ & 8.47 & [45] & [45] \\
\hline 106 & {$\left[\mathrm{C}_{6} \mathrm{mim}\right]\left[\mathrm{BF}_{4}\right]$} & methyl diethanolamine & 303.15 to 323.15 & 0.1176 to 0.903 & 45 & $\pm 3 \%$ & 6.03 & [78] & [78] \\
\hline 107 & {$\left[\mathrm{C}_{8} \mathrm{mim}\right]\left[\mathrm{NTf}_{2}\right]$} & ethanol & 298.15 & 0.1033 to 0.9041 & 11 & $\pm 0.5 \%$ & 11.16 & [79] & [79] \\
\hline 108 & {$\left[\mathrm{C}_{8} \mathrm{mim}\right]\left[\mathrm{NTf}_{2}\right]$} & methyl acetate & 298.15 & 0.1016 to 0.9301 & 11 & $\pm 0.5 \%$ & 1.96 & [49] & [49] \\
\hline 109 & {$\left[\mathrm{C}_{8} \mathrm{mim}\right]\left[\mathrm{BF}_{4}\right]$} & ethanol & 298.15 & 0.0517 to 0.9286 & 13 & $-b$ & 1.39 & [50] & [50] \\
\hline 110 & {$\left[\mathrm{C}_{8} \mathrm{mim}\right]\left[\mathrm{BF}_{4}\right]$} & butyl acetate & 298.15 & 0.05001 to 0.886 & 13 & $\pm 0.01(\mathrm{mPa} \cdot \mathrm{s})$ & 1.76 & [51] & [51] \\
\hline 111 & {$\left[\mathrm{C}_{8} \mathrm{mim}\right]\left[\mathrm{BF}_{4}\right]$} & methyl acetate & 298.15 & 0.0485 to 0.8827 & 13 & $\pm 0.01(\mathrm{mPa} \cdot \mathrm{s})$ & 5.24 & [51] & [51] \\
\hline 112 & {$\left[\mathrm{C}_{4} \mathrm{mim}\right]\left[\mathrm{PF}_{6}\right]$} & ethanol & 288.15 to 308.15 & 0.52 to 0.8 & 10 & $\pm 3 \%$ & 10.08 & [80] & $-\mathrm{a}^{\mathrm{a}}$ \\
\hline 113 & {$\left[\mathrm{C}_{2} \mathrm{mim}\right][\mathrm{DCA}]$} & water & 298.15 to 343.15 & 0.047 to 0.8701 & 40 & $0.65 \%$ & 4.92 & [59] & [59] \\
\hline 114 & {$\left[\mathrm{C}_{4}\right.$ mpyrro] $[\mathrm{DCA}]$} & $\gamma$-butyrolactone & 273.15 to 323.15 & 0.0987 to 0.9013 & 42 & $1 \%$ & 1.59 & [81] & [81] \\
\hline
\end{tabular}




\begin{tabular}{|c|c|c|c|c|c|c|c|c|c|}
\hline 115 & {$\left[\mathrm{C}_{4} \mathrm{mim}\right]\left[\mathrm{C}_{1} \mathrm{SO}_{4}\right]$} & water & 298.15 to 328.15 & 0.0534 to 0.9675 & 30 & $< \pm 0.2(\mathrm{mPa} \bullet \mathrm{s})$ & 8.82 & [82] & [82] \\
\hline 116 & {$\left[\mathrm{C}_{4} \mathrm{mim}\right]\left[\mathrm{C}_{1} \mathrm{SO}_{4}\right]$} & ethanol & 298.15 to 328.15 & 0.0555 to 0.9349 & 33 & $< \pm 0.2(\mathrm{mPa} \cdot \mathrm{s})$ & 10.20 & {$[82]$} & [82] \\
\hline 117 & {$\left[\mathrm{C}_{4} \mathrm{mim}\right][\mathrm{OTf}]$} & water & 298.15 & 0.0805 to 0.8907 & 10 & $\pm 1 \%$ & 13.36 & [29] & [29] \\
\hline 118 & {$\left[\mathrm{C}_{4} \mathrm{mim}\right][\mathrm{OAc}]$} & water & 278.15 & 0.03236 to 0.75268 & 5 & $\pm 2 \%$ & 25.84 & [71] & $-a^{a}$ \\
\hline 119 & {$\left[\mathrm{C}_{4} \mathrm{mim}\right][\mathrm{OAc}]$} & 1,2-ethanediol & 298.15 & 0.12944 to 0.78032 & 4 & $\pm 2 \%$ & 14.40 & [71] & $-a^{a}$ \\
\hline
\end{tabular}

\begin{tabular}{|c|c|c|c|c|c|c|c|c|c|}
\hline \multicolumn{10}{|c|}{ Test Set } \\
\hline 1 & {$\left[\mathrm{C}_{2} \mathrm{mim}\right]\left[\mathrm{C}_{2} \mathrm{SO}_{4}\right]$} & 2-propanol & 298.15 to 328.15 & 0.0294 to 0.8772 & 27 & $\pm 0.006(\mathrm{mPa} \bullet \mathrm{s})$ & 6.44 & [18] & [18] \\
\hline 2 & {$\left[\mathrm{C}_{2} \mathrm{mim}\right]\left[\mathrm{NTf}_{2}\right]$} & 1-propanol & 278.15 to 338.15 & 0.0519 to 0.9025 & 35 & $-c$ & 11.28 & [38] & [38] \\
\hline 3 & {$\left[\mathrm{C}_{4} \mathrm{mim}\right]\left[\mathrm{BF}_{4}\right]$} & acetone & 298.15 & 0.0508 to 0.8949 & 13 & $\pm 0.3 \%$ & 11.19 & [27] & [27] \\
\hline 4 & {$\left[\mathrm{C}_{4} \mathrm{mim}\right]\left[\mathrm{NTf} \mathrm{f}_{2}\right]$} & 1-butanol & 298.15 & 0.1382 to 0.8952 & 7 & $1.5 \%$ & 23.11 & [83] & $-a^{a}$ \\
\hline 5 & {$\left[\mathrm{C}_{4} \mathrm{mim}\right]\left[\mathrm{NTf}_{2}\right]$} & 1-propanol & 278.15 to 338.15 & 0.0508 to 0.9030 & 40 & $-c$ & 8.58 & {$[38]$} & [38] \\
\hline 6 & {$\left[\mathrm{C}_{4} \mathrm{mim}\right]\left[\mathrm{NTf} \mathrm{f}_{2}\right]$} & ethyl acetate & 298.15 & 0.1067 to 0.9478 & 11 & $\pm 0.5 \%$ & 21.42 & [77] & [77] \\
\hline 7 & {$\left[\mathrm{C}_{6} \mathrm{mim}\right]\left[\mathrm{NTf}_{2}\right]$} & ethanol & 278.15 to 338.15 & 0.047 to 0.903 & 40 & $0.1 \%$ & 5.91 & [31] & [31] \\
\hline 8 & {$\left[\mathrm{C}_{6} \mathrm{mim}\right]\left[\mathrm{BF}_{4}\right]$} & 2-methyl-1-propanol & 303.15 to 308.15 & 0.1004 to 0.8327 & 16 & $-\mathrm{b}$ & 7.82 & [84] & [84] \\
\hline 9 & {$\left[\mathrm{C}_{6} \mathrm{mim}\right]\left[\mathrm{BF}_{4}\right]$} & 1-propanol & 293.15 to 333.15 & 0.1006 to 0.8894 & 35 & $-b$ & 9.80 & [85] & [85] \\
\hline 10 & {$\left[\mathrm{C}_{6} \mathrm{mim}\right]\left[\mathrm{BF}_{4}\right]$} & 2-propanol & 293.15 to 333.15 & 0.08354 to 0.85073 & 45 & $-b$ & 14.55 & [86] & [86] \\
\hline 11 & {$\left[\mathrm{C}_{8} \mathrm{mim}\right]\left[\mathrm{NTf} \mathrm{f}_{2}\right]$} & ethyl acetate & 298.15 & 0.0984 to 0.9507 & 11 & $\pm 0.5 \%$ & 4.04 & [79] & [79] \\
\hline 12 & {$\left[\mathrm{C}_{8} \mathrm{mim}\right]\left[\mathrm{BF}_{4}\right]$} & 2-propanol & 298.15 & 0.0459 to 0.8697 & 13 & $-b$ & 7.11 & {$[50]$} & [50] \\
\hline 13 & {$\left[\mathrm{C}_{4} \mathrm{mim}\right]\left[\mathrm{PF}_{6}\right]$} & acetone & 298.15 & 0.0519 to 0.8853 & 13 & $\pm 0.2 \%$ & 7.55 & [52] & [52] \\
\hline 14 & {$\left[\mathrm{C}_{4} \mathrm{mim}\right]\left[\mathrm{PF}_{6}\right]$} & 3-pentanone & 298.15 & 0.052 to 0.8912 & 13 & $\pm 0.2 \%$ & 7.93 & [52] & [52] \\
\hline 15 & {$\left[\mathrm{C}_{4} \mathrm{mim}\right]\left[\mathrm{PF}_{6}\right]$} & ethyl acetate & 298.15 & 0.0496 to 0.9063 & 13 & $\pm 0.2 \%$ & 15.84 & {$[52]$} & [52] \\
\hline 16 & {$\left[\mathrm{C}_{4} \mathrm{mim}\right][\mathrm{SCN}]$} & 1-heptanol & 298.15 to 348.15 & 0.0806 to 0.9793 & 66 & $<1 \%$ & 4.71 & [67] & [67] \\
\hline
\end{tabular}




\begin{tabular}{|c|c|c|c|c|c|c|c|c|c|}
\hline 17 & {$\left[\mathrm{C}_{4} \mathrm{mim}\right][\mathrm{SCN}]$} & 1-pentanol & 298.15 to 348.15 & 0.0865 to 0.9807 & 66 & $<0.05 \%$ & 5.73 & [66] & [66] \\
\hline 18 & {$\left[\mathrm{C}_{4} \mathrm{mim}\right][\mathrm{SCN}]$} & 1-butanol & 298.15 to 348.15 & 0.0262 to 0.9089 & 60 & $<0.05 \%$ & 5.39 & {$[66]$} & {$[66]$} \\
\hline 19 & {$\left[\mathrm{C}_{4} \mathrm{mim}\right][\mathrm{SCN}]$} & 1-propanol & 298.15 to 348.15 & 0.0267 to 0.9342 & 60 & $<1 \%$ & 6.38 & {$[65]$} & {$[65]$} \\
\hline 20 & {$\left[\mathrm{C}_{4} \mathrm{mim}\right][\mathrm{SCN}]$} & 1-octanol & 298.15 to 348.15 & 0.0874 to 0.9619 & 54 & $<1 \%$ & 4.62 & [67] & [67] \\
\hline 21 & {$\left[\mathrm{C}_{4} \mathrm{mim}\right][\mathrm{SCN}]$} & 1-nonanol & 298.15 to 348.15 & 0.1203 to 0.9651 & 48 & $<1 \%$ & 3.81 & [67] & [67] \\
\hline 22 & {$\left[\mathrm{~N}_{1114}\right]\left[\mathrm{NTf}_{2}\right]$} & 1,2-propanediol & 323.15 to 353.15 & 0.1157 to 0.868 & 49 & $\pm 0.1 \%$ & 6.00 & [73] & [73] \\
\hline 23 & {$\left[\mathrm{C}_{8} \mathrm{mim}\right]\left[\mathrm{BF}_{4}\right]$} & propyl acetate & 298.15 & 0.0471 to 0.8994 & 13 & $\pm 0.01(\mathrm{mPa} \cdot \mathrm{s})$ & 8.56 & [51] & [51] \\
\hline 24 & {$\left[\mathrm{C}_{8} \mathrm{mim}\right] \mathrm{Cl}$} & 1-propanol & 298.15 to 328.15 & 0.0271 to 0.8929 & 33 & $<1(\mathrm{mPa} \cdot \mathrm{s})$ & 12.99 & [75] & [75] \\
\hline
\end{tabular}


2.2 Functional Groups. In our previous studies, the UNIFAC-VISCO model was developed to estimate the viscosity of pure ILs where each ion was regarded as an individual functional group $[14,15]$. In the present study, a similar approach was used to define the IL-viscosity contribution for investigated mixtures. In terms of the organic component in the mixture, the original cleavage reported by Chevalier et al. [87] was also used in the current study. The cleavages are identical for the branched and linear hydrocarbons with the same number of carbon atoms, which limits the number of organic functional groups [87]. Although isomers of organic components are not characterized by using this definition, ILs with different alkyl chain shapes (branched and linear) could be evaluated thanks to the 3D structure of ions contributing to the volume and surface area values ( $R$ and $Q)[14]$.

Chevalier et al. [88] reported that methanol could not be split into the groups $\mathrm{CH}_{3}$ and $\mathrm{OH}$ like other alcohols, due to a peculiarity. Several molecular solvents also show a similar characteristic and are defined as the single groups, such as water, chloroform, dichloromethane, pyridine, and thiophene. Table 2 shows the list of organic components and their corresponding functional groups used during this work. 
2.3 Proposed Model. The UNIFAC-VISCO model is a group contribution model developed by Chevalier et al. $[87,88]$ to predict the viscosity of the liquid mixtures. Briefly, the viscosity of a given mixture is calculated as follows:

$\ln (\mu)=\sum_{i=1}^{C} x_{i} \ln \left(\mu_{i} \cdot \frac{V_{i}}{V_{m}}\right)+\frac{g_{c}^{E}}{R T}-\frac{g_{r}^{E}}{R T}$

where, $\mu$ is the viscosity of the mixture; subscript $i$ represents the pure component in the mixture (i.e., the IL and the solvent); $C$ is the total number of the pure components existing in the mixture, and equals 2 because only binary mixtures were investigated in this paper. $x_{i}$ is the mole fraction of component $i ; \mu_{i}$ is the purecomponent viscosity of component $i ; V_{i}$ is the pure-component molar volume of component $i ; V_{m}$ is the ideal molar volume of the mixture calculated by using the following equation:

$V_{m}=\sum_{i=1}^{C} x_{i} V_{i}$

The UNIFAC-VISCO combinatorial term reported in the eq. 1 is defined by:

$\frac{g_{C}^{E}}{R T}=\sum_{i=1}^{C} x_{i} \ln \frac{\phi_{i}}{x_{i}}+5 \sum_{i=1}^{C} x_{i} q_{i} \ln \frac{\theta_{i}}{\phi_{i}}$

where

$\theta_{i}=\frac{x_{i} q_{i}}{\sum_{j} x_{j} q_{j}}$

$\phi_{i}=\frac{x_{i} r_{i}}{\sum_{j} x_{j} r_{j}}$

$q_{i}=\sum_{k=1}^{N} n_{i, k} Q_{k}$

$r_{i}=\sum_{k=1}^{N} n_{i, k} R_{k}$

The volume $R$ and surface area $Q$ of ionic groups were obtained by using the COSMOthermX software (version C30_1601) as reported by our group previously $[14,15,89]$. $R$ and $Q$ values of organic groups are collected from values reported by 
Fredenslund et al. [90,91]. $R$ and $Q$ values of all the functional groups are presented in the Table S2 of the Supporting Information.

The UNIFAC-VISCO residual term reported in the eq. 1 is defined by:

$\frac{g_{r}^{E}}{R T}=\sum_{i=1}^{C} x_{i}\left[\sum_{m=1}^{N} n_{m, i}\left(\ln \gamma_{m}-\ln \gamma_{m, i}\right)\right]$

where $N$ is the total number of the functional groups existing in the binary system; $n_{m}$ and $\ln \gamma_{m}$ refer to the group fractions in the mixture, and $n_{m, i}$ and $\ln \gamma_{m, i}$ refer to the group fractions in pure component $i . \ln \gamma_{m}$ is defined as:

$\ln \gamma_{m}=Q_{m}\left[1-\ln \left(\sum_{i=1}^{N} \Theta_{i} \Psi_{i, m}\right)-\sum_{i=1}^{N} \frac{\Theta_{i} \Psi_{m, i}}{\sum_{j=1}^{N} \Theta_{j} \Psi_{j, i}}\right]$

The $\Theta$ value is obtained by using eq 10 :

$\Theta_{m}=\frac{x_{m} Q_{m}}{\sum_{i=1}^{N} X_{i} Q_{i}}, m=1,2,3 \ldots N$

The group interaction parameters $\Psi_{m n}$ are calculated as follows:

$\Psi_{m n}=\exp \left(-\frac{\alpha_{m n}}{298.15}\right)$

where $\alpha_{m n}$ are the group interaction potential energy parameters between the defined functional groups $m$ and $n$.

2.4 Determination of The Interaction Parameters $\boldsymbol{\alpha}_{m n}$. A set of interaction parameters $\alpha_{m n}$ is required for the calculation of the viscosity of binary mixtures using the UNIFAC-VISCO model. The values of interaction parameters between ionic groups $\left(\alpha_{\text {ion/ion }}\right)$ were collected from our previous paper [14]. The values of interaction parameters between some organic groups ( $\left.\alpha_{\text {organic/organic }}\right)$ were collected from Chevalier et al. [88]. The other unknown interaction parameters between organic and ionic groups ( $\alpha_{\text {ion/organic }}$ and $\left.\alpha_{\text {organic/organic }}\right)$ were regressed by using the experimental training datasets. To obtain the values of $\alpha_{m n}$, we 
performed the Marquardt [92] optimization of the following objective function:

$O F=\frac{1}{M} \sum_{i=1}^{M}\left(\frac{\mu_{\text {exp }}-\mu_{\text {cal }}}{\mu_{\text {exp }}}\right)^{2} \rightarrow \min$

where $M$ is the number of data points; $\mu_{\text {exp }}$ and $\mu_{c a l}$ are the experimental and calculated viscosity data, respectively.

To carry out the regression, the 'Isqnonlin' function of the MATLAB Optimization Toolbox was used. In this work, 3365 training data were regressed altogether to obtain the global optimal values of interaction parameters. 


\section{RESULTS AND DISCUSSION}

All the binary interaction parameters used in this work are presented in the Table S3 of the Supporting Information. The regression result was characterized by calculating the relative absolute average deviation (RAAD\%, eq. 13) between the calculated ( $\left.\mu_{\text {cal }}\right)$ and experimental viscosity $\left(\mu_{\text {exp }}\right)$ data.

$R A A D \%=100 \times \frac{1}{M} \sum_{i=0}^{M}\left|\frac{\mu_{e x p}-\mu_{c a l}}{\mu_{e x p}}\right|$

The deviation between each experimental viscosity dataset and correlated (training set; overall RAAD\% close to $5.0 \%$ ) for each investigated binary system used to determine the missing UNIFAC-VISCO parameters is reported in Table 1. As shown in Table 1 and in Figure 1, the quality of the developed UNIFAC-VISCO model was then assessed by comparing experimental data with those calculated for wide ranges of temperature, composition, and chemical structures leading to large values of viscosity up to $14000 \mathrm{mPa} \cdot \mathrm{s}$. As illustrated in Figures 1 and 2, the correlated results show, generally, a good agreement with the experimental data, except in two cases: i.e. the ([ $\left.\mathrm{C}_{6} \mathrm{mim}\right]\left[\mathrm{BF}_{4}\right]+$ propylamine) viscosity dataset reported by Kermanpour et al. [43] and the $\left(\left[\mathrm{C}_{4} \mathrm{mim}\right][\mathrm{OAc}]+\right.$ water $)$ dataset published by Fendt et al. [71] for which a deviation higher than $20 \%$ is observed in both cases.

To further assess the UNIFAC-VISCO model, 781 test data points for 24 binary mixtures, not originally used during the training set, were then compared to the evaluated viscosity data by using the interaction parameters estimated by using the training set. 
An arbitrary binary mixture with unknown viscosity could also be estimated thanks to the optimized values of the binary interaction parameters between the functional groups, constituting the given mixture. The evaluation result for each binary system is presented in Table 1 and is also shown in Figures 1-2. The overall RAAD\% for the test set is close to $7.7 \%$ (see Table 1 and Figure 2), which demonstrates that the interaction parameters estimated during this work are accurate enough to evaluate then the viscosity data for original mixtures.

Nevertheless, as shown in Figure 2, a correlated data point for the $\left(\left[\mathrm{C}_{4} \mathrm{mim}\right][\mathrm{OAc}]+\right.$ water) binary system presents a relative deviation higher than $60 \%$. However, only one research group reported the experimental viscosity data for the system ([ $\left.\mathrm{C}_{4} \mathrm{mim}\right][\mathrm{OAc}]$ + water), to date [71]. The viscosity of the pure [ $\left.\mathrm{C}_{4} \mathrm{mim}\right][\mathrm{OAc}]$ measured by Fendt et al. [71] is close to $485 \mathrm{mPa} \cdot \mathrm{s}$ at $298.15 \mathrm{~K}$; while by looking at the literature, it is obvious to highlight a clear discrepancy between experimental viscosity values reported for the [ $\mathrm{C}_{4} \mathrm{mim}$ ][OAc] at $298.15 \mathrm{~K}$; e.g. $297 \mathrm{mPa} \cdot \mathrm{s}$ [93], $440 \mathrm{mPa} \cdot \mathrm{s}$ [94], $485.1 \mathrm{mPa} \cdot \mathrm{s}$ [95], and $485 \mathrm{mPa} \cdot \mathrm{s}$ [71]. As reported in our previous paper [14], a viscosity dataset [96] was recommended through the gnostic analysis of five viscosity datasets [71,93-96] for $\left[\mathrm{C}_{4} \mathrm{mim}\right][\mathrm{OAc}]$ over a temperature range from (283.15 to 393.15$) \mathrm{K}$. The interaction parameters between ions $\left[\mathrm{C}_{4} \mathrm{mim}\right]^{+}$and $[\mathrm{OAc}]^{-}$were then calculated by regressing the recommended dataset [96], leading to an estimated viscosity value close to 424.6 $\mathrm{mPa} \cdot \mathrm{s}$ at $298.15 \mathrm{~K}$ for the pure component [ $\left.\mathrm{C}_{4} \mathrm{mim}\right][\mathrm{OAc}]$ by using the UNIFAC-VISCO method [14]. Therefore, such a discrepancy on the pure component viscosity values definitely contributes to increasing errors during the estimation of the viscosity for the 
$\left(\left[\mathrm{C}_{4} \mathrm{mim}\right][\mathrm{OAc}]+\right.$ water $)$ binary system as shown in Figure 2.

Table 3 shows the estimation results (including correlated and evaluated data) for several main types of molecular solvents to further analyze the effect of the molecular solvent structure on the predictive capability of the UNIFAC-VISCO model. The deviation between the experimental viscosity data and those determined using the UNIFAC-VISCO model follows the order: water $>$ amines $>$ alcohols $>$ esters $>$ ketones $>$ amides. The binary systems with water show the poorest estimation result. This trend could be attributed to the complexity of $\left(\mathrm{IL}+\mathrm{H}_{2} \mathrm{O}\right)$ systems, driven by strong solvention interactions, like H-bonds, between water and IL, which may induce experimental (hydrolysis reaction [97], trace of water originally present in the pure IL, etc.) and computational errors. The difference between the experimental viscosity values for pure ILs used in this work for the calculation of the viscosity data of selected mixtures and those used in our previous paper [14] to determine the interaction parameters $\alpha_{\text {ion/ion }}$ also contribute to an increase in the observed errors of the UNIFAC-VISCO model for some IL-based mixtures. In other words, the quality of the proposed method is strongly affected by the overall accuracy of data reported in the literature as expected for a such complex property like the viscosity.

Furthermore, the impact of the non-ideality of the volumetric properties of the solution on the quality of the proposed method was also exemplified by using the $\left(\left[\mathrm{C}_{2} \mathrm{mim}\right]\left[\mathrm{C}_{2} \mathrm{SO}_{4}\right]+1\right.$-propanol) binary system. The experimental viscosities, densities, and excess molar volumes of this selected system are collected from González et al. [18] and provided in Table S4 of the Supporting Information, together with the 
calculated binary viscosities by implementing the ideal and then the real molar volumes into the UNIFAC-VISCO model, respectively. Figure 3 shows the excess molar volumes of the system $\left(\left[\mathrm{C}_{2} \mathrm{mim}\right]\left[\mathrm{C}_{2} \mathrm{SO}_{4}\right]+1\right.$-propanol), along with the fitted curves by using the Redlich-Kister [98] equation (Eq. 14) as a function of the mole fraction of solvent.

$V^{E}=x_{s}\left(1-x_{s}\right) \sum_{p=0}^{M} B_{p}\left(2 x_{s}-1\right)^{P}$

where $V^{E}$ is the excess molar volume; $x_{s}$ is the mole fraction of solvent; $B_{p}$ are the Redlich-Kister fitting parameters; and $M$ is the degree of the polynomial expansion. The fitting parameters reported by González et al. [18] are presented in Table S5 of the Supporting Information.

Figure 4 shows the difference on the calculated viscosities induced by using the nonideality of the system $\left(\left[\mathrm{C}_{2} \mathrm{mim}\right]\left[\mathrm{C}_{2} \mathrm{SO}_{4}\right]+1\right.$-propanol). The minimum of $V^{E}$ presents at approximately $x_{s}=0.6$, as shown in Figure 3 . While the largest difference between the calculated viscosities by two different molar volumes is present at nearly 0.8 mole fraction of 1-propanol. The negative excess molar volume decreases as the temperature increases. Analogous temperature dependence behavior was observed for the calculated viscosities difference in Figure 4. However, the minimum difference between the calculated viscosities is better than $-0.6 \%$ for the system ([ $\left.\mathrm{C}_{2} \mathrm{mim}\right]\left[\mathrm{C}_{2} \mathrm{SO}_{4}\right]+$ 1-propanol). The RAAD\% between the experimental viscosities and the calculated viscosities assuming the system to be ideal is $3.62 \%$, and the RAAD\% between the experimental viscosities and the calculated values considering their nonideality is $3.55 \%$. Hence, only a global $0.07 \%$ deviation was caused by the non-ideality 
of the binary system. Therefore, it is reasonable and acceptable to assume all binary systems investigated in this work as ideal. 


\section{CONCLUSIONS}

In this work, the experimental viscosity values of (IL + molecular solvent) binary mixtures were estimated by using the modified UNIFAC-VISCO model. During this work, 3365 experimental viscosity data for 119 different binary systems were regressed altogether to determine missing interaction parameters between ions and molecular solvent group parameters. Then, 781 experimental data of 24 binary systems, not originally included in the training set, were used to assess the performance of the established model. The overall estimation result is close to $5.5 \%$, amongst $5 \%$ for the training result and $7.7 \%$ for the test result, which proves that the UNIFAC-VISCO-based method is able to correlate and then evaluate the viscosity of (ILS + molecular solvent) binary mixtures as the function of temperature and composition at $0.1 \mathrm{MPa}$. 


\section{ASSOCIATED CONTENT}

\section{Supporting Information}

The volumetric parameters for selected cations and anions are provided in Table S1. $R$ and $Q$ values of the functional groups, and the interaction parameters $\alpha_{m n}$ used in this work are presented in Tables S2-S3. Experimental density, viscosity, and excess molar volume data, along with calculated viscosity values for the system $\left(\left[\mathrm{C}_{2} \mathrm{mim}\right]\left[\mathrm{C}_{2} \mathrm{SO}_{4}\right]+\right.$ 1-propanol) are given in Table S4. Parameters fitted to the RedlichKister equation for the excess molar volume of $\left(\left[\mathrm{C}_{2} \mathrm{mim}\right]\left[\mathrm{C}_{2} \mathrm{SO}_{4}\right]\right.$ and 1-propanol) are provided in Table S5.

\section{AUTHOR INFORMATION}

\section{Corresponding Author}

*E-mail: ji@univ-tours.fr or johan.jacquemin@qub.ac.uk

\section{Funding}

N.Z. and J.J. acknowledge gratefully the CEA le Ripault (Grant No 4600261677/P6E31) and the Engineering and Physical Sciences Research Council (EPSRC) for supporting this work financially (EPSRC First Grant Scheme, Grant No EP/M021785/1).

\section{Notes}

The authors declare no competing financial interest. 


\section{ABBREVIATIONS}

UNIQUAC UN/versal QUasichemical Activity Coefficient

UNIFAC-VISCO UNIversal Functional-group Activity Coefficient-VISCOsity

NRTL Non-Random Two-Liquid

MTSM Modified Two-Suffix-Margules

ASOG-VISCO Analytical Solution Of Groups-VISCOsity

\section{Cations:}

$[\mathrm{mim}]^{+}$methylimidazolium

$\left[\mathrm{C}_{1} \text { mim }\right]^{+}$1,3-dimethylimidazolium

$\left[\mathrm{C}_{2} \mathrm{mim}\right]^{+}$1-ethyl-3-methylimidazolium

$\left[\mathrm{C}_{3} \mathrm{mmim}\right]^{+}$1-propyl-2,3-dimethylimidazolium

$\left[\mathrm{C}_{4} \text { mim }\right]^{+}$1-butyl-3-methylimidazolium

$\left[\mathrm{C}_{6} \mathrm{mim}\right]^{+}$1-hexyl-3-methylimidazolium

$\left[\mathrm{C}_{8} \mathrm{mim}\right]^{+}$1-octyl-3-methylimidazolium

$\left[\mathrm{C}_{4} \mathrm{mmim}\right]^{+}$1-butyl-2,3-dimethylimidazolium

$\left[\mathrm{C}_{4} \mathrm{py}\right]^{+}$1-butylpyridinium

$\left[\mathrm{C}_{4} \text { mpyrro }\right]^{+}$1-butyl-1-methylpyrrolidinium

$\left[\mathrm{N}_{1114}\right]^{+}$butyl-trimethyl-ammonium

$\left[\mathrm{P}_{66614}\right]^{+}$trihexyl(tetradecyl)phosphonium

Anions:

$\left[\mathrm{BF}_{4}\right]^{-}$tetrafluoroborate

$\left[\mathrm{NO}_{3}\right]^{-}$nitrate 
$\left[\mathrm{NTf}_{2}\right]^{-}$bis(trifluoromethylsulfonyl)imide

$\left[\mathrm{C}_{1} \mathrm{SO}_{4}\right]^{-}$methylsulfate

$\left[\mathrm{C}_{2} \mathrm{SO}_{4}\right]^{-}$ethylsulfate

$\left[\mathrm{PF}_{6}\right]^{-}$hexafluorophosphate

$[D C A]^{-}$dicyanamide

$[\mathrm{SCN}]^{-}$thiocyanate

$[\mathrm{OAc}]^{-}$acetate

[OTf] ${ }^{-}$triflate

$\mathrm{Cl}^{-}$chloride

\section{Nomenclature}

\section{Roman Letters:}

$a$ parameter in Seddon equation

$C$ total number of pure components in UNIFAC-VISCO method

$g_{c}^{E}$ combinatorial term in UNIFAC-VISCO method

$g_{r}^{E}$ residual term in UNIFAC-VISCO method

$n_{i, k}$ total number of $k^{\text {th }}$ group present in component $i$

$M$ number of data points

$N$ total number of groups

$q_{i}$ van der Waals' surface area of component $i$

$Q_{k}$ group surface area parameter

$r_{i}$ van der Waals' volume of component $i$

$R_{k}$ group volume parameter. 
$\mathcal{R}$ gas constant $(\mathrm{J} / \mathrm{mol} \cdot \mathrm{K})$

$T$ temperature (K)

$V_{i}$ pure-component molar volume $\left(\mathrm{m}^{3} / \mathrm{kmol}\right)$

$V_{m}$ mixture molar volume $\left(\mathrm{m}^{3} / \mathrm{kmol}\right)$

$x_{i}$ mole fraction of the component $i$

$x_{s}$ mole fraction of the molecular solvent

\section{Greek Letters:}

$\alpha_{m n}$ group interaction parameter between groups $m$ and $n$

$\gamma_{m}$ residual activity coefficient

$\rho_{I L}$ density of pure ionic liquid $\left(\mathrm{g} \cdot \mathrm{cm}^{-3}\right)$

$\theta_{i}$ molecular surface area fraction of component $i$

$\Theta_{i}$ area fraction for group $i$

$\mu$ viscosity of mixture (mPa.s)

$\mu_{i}$ viscosity of pure component $i$

$\mu_{I L}$ viscosity of pure ionic liquid

$\mu_{\text {exp }}$ viscosity experimentally measured

$\mu_{\text {cal }}$ viscosity calculated by our method

$\mu_{\text {corre }}$ viscosity correlated by the Seddon equation

$\phi_{i}$ molecular volume fraction of component $i$

$\Psi_{m, i}$ group interaction parameter 


\section{REFERENCES}

[1] O. Ciocirlan, O. Croitoru, O. Iulian, Viscosity of binary mixtures of 1-ethyl-3methylimidazolium tetrafluoroborate ionic liquid with four organic solvents, J. Chem. Thermodyn. 101 (2016) 285-292.

[2] Y. Wang, D. Chen, X. OuYang, Viscosity Calculations for lonic Liquid-Cosolvent Mixtures Based on Eyring's Absolute Rate Theory and Activity Coefficient Models, 55 (2010) 4878-4884.

[3] K.R. Seddon, A. Stark, M.-J. Torres, Influence of chloride, water, and organic solvents on the physical properties of ionic liquids, Pure Appl. Chem. 72 (2000) $2275-2287$.

[4] A. Ruiz-Llamas, R. Macías-Salinas, Modeling the Dynamic Viscosity of Ionic Solutions, Ind. Eng. Chem. Res. 54 (2015) 7169-7179.

[5] Y.-C. He, X.-J. Xu, L.-J. Yang, B. Ding, Viscosity modeling for ionic liquid solutions by Eyring-Wilson equation, Chem. Ind. Chem. Eng. Q. 18 (2012) 441-447.

[6] D.M. Bajić, S.P. Šerbanović, E.M. Živković, J. Jovanović, M.L. Kijevčanin, Prediction and correlation of viscosity of binary mixtures of ionic liquids with organic solvents, J. Mol. Liq. 197 (2014) 1-6.

[7] S. Fang, C.-H. He, A new one parameter viscosity model for binary mixtures, AIChE J. 57 (2011) 517-524.

[8] S. Atashrouz, M. Zarghampour, S. Abdolrahimi, G. Pazuki, B. Nasernejad, Estimation of the Viscosity of Ionic Liquids Containing Binary Mixtures Based on the Eyring's Theory and a Modified Gibbs Energy Model, J. Chem. Eng. Data. 59 
(2014) 3691-3704.

[9] B.E. Poling, J.M. Prausnitz, J.P. O'Connell, The properties of gases and liquids, 5th ed., McGraw-Hill: New York, 2001.

[10] R.A. McAllister, The viscosity of liquid mixtures, AIChE J. 6 (1960) 427-431.

[11] R.J. Martins, M.J.E. de M. Cardoso, O.E. Barcia, Excess Gibbs Free Energy Model for Calculating the Viscosity of Binary Liquid Mixtures, Ind. Eng. Chem. Res. 39 (2000) 849-854.

[12] L.T. Novak, Relationship between the Intrinsic Viscosity and Eyring-NRTL Viscosity Model Parameters, Ind. Eng. Chem. Res. 43 (2004) 2602-2604.

[13] R. Sadeghi, Segment-based Eyring-Wilson viscosity model for polymer solutions, J. Chem. Thermodyn. 37 (2005) 445-448.

[14] N. Zhao, R. Oozeerally, V. Degirmenci, Z. Wagner, M. Bendová, J. Jacquemin, New Method Based on the UNIFAC-VISCO Model for the Estimation of Ionic Liquids Viscosity Using the Experimental Data Recommended by Mathematical Gnostics, J. Chem. Eng. Data. 61 (2016) 3908-3921.

[15] N. Zhao, J. Jacquemin, R. Oozeerally, V. Degirmenci, New Method for the Estimation of Viscosity of Pure and Mixtures of Ionic Liquids Based on the UNIFAC-VISCO Model, J. Chem. Eng. Data. 61 (2016) 2160-2169.

[16] J. Jacquemin, R. Ge, P. Nancarrow, D.W. Rooney, M.F. Costa Gomes, A.A.H. Pádua, C. Hardacre, Prediction of Ionic Liquid Properties. I. Volumetric Properties as a Function of Temperature at $0.1 \mathrm{MPa}$, J. Chem. Eng. Data. 53 (2008) 716-726. 
[17] J. Jacquemin, P. Nancarrow, D.W. Rooney, M.F. Costa Gomes, P. Husson, V. Majer, A.A.H. Pádua, C. Hardacre, Prediction of Ionic Liquid Properties. II. Volumetric Properties as a Function of Temperature and Pressure, J. Chem. Eng. Data. 53 (2008) 2133-2143.

[18] E.J. González, B. González, N. Calvar, Á. Domínguez, Physical Properties of Binary Mixtures of the Ionic Liquid 1-Ethyl-3-methylimidazolium Ethyl Sulfate with Several Alcohols at $T=(298.15,313.15$, and 328.15) $\mathrm{K}$ and Atmospheric Pressure, J. Chem. Eng. Data. 52 (2007) 1641-1648.

[19] E. Gómez, B. González, N. Calvar, E. Tojo, Á. Domínguez, Physical Properties of Pure 1-Ethyl-3-methylimidazolium Ethylsulfate and Its Binary Mixtures with Ethanol and Water at Several Temperatures, J. Chem. Eng. Data. 51 (2006) 2096-2102.

[20] E. Vercher, F.J. Llopis, V. González-Alfaro, P.J. Miguel, V. Orchillés, A. MartínezAndreu, Volumetric properties, viscosities and refractive indices of binary liquid mixtures of tetrafluoroborate-based ionic liquids with methanol at several temperatures, J. Chem. Thermodyn. 90 (2015) 174-184.

[21] E. Rilo, J. Vila, M. García, L.M. Varela, O. Cabeza, Viscosity and Electrical Conductivity of Binary Mixtures of $\mathrm{C}_{n} \mathrm{MIM}-\mathrm{BF}_{4}$ with Ethanol at $288 \mathrm{~K}, 298 \mathrm{~K}, 308$ K, and 318 K, J. Chem. Eng. Data. 55 (2010) 5156-5163.

[22] X.-H. Fan, Y.-P. Chen, C.-S. Su, Density and Viscosity Measurements for Binary Mixtures of 1-Ethyl-3-methylimidazolium Tetrafluoroborate ([Emim] $\left.\left[\mathrm{BF}_{4}\right]\right)$ with Dimethylacetamide, Dimethylformamide, and Dimethyl Sulfoxide, J. Chem. Eng. 
Data. 61 (2016) 920-927.

[23] Q. Zhou, L.-S. Wang, H.-P. Chen, Densities and Viscosities of 1-Butyl-3methylimidazolium Tetrafluoroborate $+\mathrm{H}_{2} \mathrm{O}$ Binary Mixtures from (303.15 to 353.15) K, J. Chem. Eng. Data. 51 (2006) 905-908.

[24] J.-Y. Wu, Y.-P. Chen, C.-S. Su, Density and Viscosity of Ionic Liquid Binary Mixtures of 1-n-Butyl-3-methylimidazolium Tetrafluoroborate with Acetonitrile, N,N-Dimethylacetamide, Methanol, and N-Methyl-2-pyrrolidone, J. Solution Chem. 44 (2015) 395-412.

[25] O. Ciocirlan, O. Croitoru, O. Iulian, Densities and Viscosities for Binary Mixtures of 1-Butyl-3-Methylimidazolium Tetrafluoroborate Ionic Liquid with Molecular Solvents, J. Chem. Eng. Data. 56 (2011) 1526-1534.

[26] J. Wang, Y. Tian, Y. Zhao, K. Zhuo, A volumetric and viscosity study for the mixtures of 1-n-butyl-3-methylimidazolium tetrafluoroborate ionic liquid with acetonitrile, dichloromethane, 2-butanone and N, N-dimethylformamide, Green Chem. 5 (2003) 618-622.

[27] Y. Tian, X. Wang, J. Wang, Densities and Viscosities of 1-Butyl-3methylimidazolium Tetrafluoroborate + Molecular Solvent Binary Mixtures, J. Chem. Eng. Data. 53 (2008) 2056-2059.

[28] Y. Zhao, X. Zhang, S. Zeng, Q. Zhou, H. Dong, X. Tian, S. Zhang, Density, Viscosity, and Performances of Carbon Dioxide Capture in 16 Absorbents of Amine + Ionic Liquid $+\mathrm{H}_{2} \mathrm{O}$, Ionic Liquid $+\mathrm{H}_{2} \mathrm{O}$, and Amine $+\mathrm{H}_{2} \mathrm{O}$ Systems, J. Chem. Eng. Data. 55 (2010) 3513-3519. 
[29] W. Li, Z. Zhang, B. Han, S. Hu, Y. Xie, G. Yang, Effect of Water and Organic Solvents on the Ionic Dissociation of Ionic Liquids, J. Phys. Chem. B. 111 (2007) $6452-6456$.

[30] S. Tian, Y. Hou, W. Wu, S. Ren, K. Pang, Physical Properties of 1-Butyl-3methylimidazolium Tetrafluoroborate/N-Methyl-2-pyrrolidone Mixtures and the Solubility of $\mathrm{CO}_{2}$ in the System at Elevated Pressures, J. Chem. Eng. Data. 57 (2012) 756-763.

[31] R. Salinas, J. Pla-Franco, E. Lladosa, J.B. Montón, Density, Speed of Sound, Viscosity, and Excess Properties of Binary Mixtures Formed by Ethanol and Bis(trifluorosulfonyl)imide-Based lonic Liquids, J. Chem. Eng. Data. 60 (2015) $525-540$.

[32] H. Yao, S. Zhang, J. Wang, Q. Zhou, H. Dong, X. Zhang, Densities and Viscosities of the Binary Mixtures of 1-Ethyl-3-methylimidazolium Bis(trifluoromethylsulfonyl)imide with N-Methyl-2-pyrrolidone or Ethanol at T $=(293.15$ to 323.15$)$ K, J. Chem. Eng. Data. 57 (2012) 875-881.

[33] M.L.S. Batista, L.I.N. Tomé, C.M.S.S. Neves, J.R.B. Gomes, J.A.P. Coutinho, Characterization of systems of thiophene and benzene with ionic liquids, J. Mol. Liq. 192 (2014) 26-31.

[34] M. Vraneš, S. Papović, A. Tot, N. Zec, S. Gadžurić, Density, excess properties, electrical conductivity and viscosity of 1-butyl-3-methylimidazolium bis(trifluoromethylsulfonyl)imide+ + -butyrolactone binary mixtures, J. Chem. Thermodyn. 76 (2014) 161-171. 
[35] J. Salgado, T. Regueira, L. Lugo, J. Vijande, J. Fernández, J. García, Density and viscosity of three (2,2,2-trifluoroethanol + 1-butyl-3-methylimidazolium) ionic liquid binary systems, J. Chem. Thermodyn. 70 (2014) 101-110.

[36] A.E. Andreatta, M. Francisco, E. Rodil, A. Soto, A. Arce, Isobaric vapour-liquid equilibria and physical properties for isopropyl acetate + isopropanol + 1-butyl3-methyl-imidazolium bis(trifluoromethylsulfonyl)imide mixtures, Fluid Phase Equilib. 300 (2011) 162-171.

[37] M.M. Akbar, T. Murugesan, Thermophysical properties for the binary mixtures of 1-hexyl-3-methylimidazolium bis(trifluoromethylsulfonyl)imide [hmim] $\left[\mathrm{Tf}_{2} \mathrm{~N}\right]$ + N-methyldiethanolamine (MDEA) at temperatures (303.15 to 323.15$) \mathrm{K}, \mathrm{J}$. Mol. Liq. 169 (2012) 95-101.

[38] R. Salinas, J. Pla-Franco, E. Lladosa, J.B. Montón, Density, Viscosity, and Sound Speed of Bis(trifluoromethylsulfonyl)imide-Based lonic Liquids + 1-Propanol Mixtures, J. Chem. Eng. Data. 61 (2016) 56-66.

[39] A. Haghani, D.R. Saeva, H. Iloukhani, M.M. Hoffmann, Comparing Compositionand Temperature-Dependent Viscosities of Binary Systems Involving Ionic Liquids, J. Chem. Eng. Data. 60 (2015) 3272-3288.

[40] A. Ahosseini, L.R. Weatherley, A.M. Scurto, Viscosity and Diffusivity for the lonic Liquid 1-Hexyl-3-methyl-imidazolium Bis(trifluoromethylsulfonyl)amide with 1Octene, J. Chem. Eng. Data. 56 (2011) 3715-3721.

[41] A. Ahosseini, B. Sensenich, L.R. Weatherley, A.M. Scurto, Phase Equilibrium, Volumetric, and Interfacial Properties of the Ionic Liquid, 1-Hexyl-3- 
methylimidazolium Bis(trifluoromethylsulfonyl)amide and 1-Octene, J. Chem. Eng. Data. 55 (2010) 1611-1617.

[42] G. Vakili-Nezhaad, M. Vatani, M. Asghari, I. Ashour, Effect of temperature on the physical properties of 1-butyl-3-methylimidazolium based ionic liquids with thiocyanate and tetrafluoroborate anions, and 1-hexyl-3-methylimidazolium with tetrafluoroborate and hexafluorophosphate anions, J. Chem. Thermodyn. 54 (2012) 148-154.

[43] F. Kermanpour, H. Iloukhani, M. Javanshad, Measurement and modeling the excess properties of binary and ternary mixtures containing $[\mathrm{Hmim}]\left[\mathrm{BF}_{4}\right], 2-$ methyl-2-propanol, and propylamin compounds at 298.15K using PFP theory, J. Mol. Liq. 188 (2013) 22-27.

[44] E. Rilo, J. Vila, J. Pico, S. García-Garabal, L. Segade, L.M. Varela, O. Cabeza, Electrical Conductivity and Viscosity of Aqueous Binary Mixtures of 1-Alkyl-3methyl Imidazolium Tetrafluoroborate at Four Temperatures, J. Chem. Eng. Data. 55 (2010) 639-644.

[45] A. Zhu, J. Wang, R. Liu, A volumetric and viscosity study for the binary mixtures of 1-hexyl-3-methylimidazolium tetrafluoroborate with some molecular solvents, J. Chem. Thermodyn. 43 (2011) 796-799.

[46] F.S. Oliveira, M.G. Freire, P.J. Carvalho, J.A.P. Coutinho, J.N.C. Lopes, L.P.N. Rebelo, I.M. Marrucho, Structural and Positional Isomerism Influence in the Physical Properties of Pyridinium NTf 2 -Based lonic Liquids: Pure and WaterSaturated Mixtures, J. Chem. Eng. Data. 55 (2010) 4514-4520. 
[47] N.D. Khupse, A. Kumar, Dramatic Change in Viscosities of Pure lonic Liquids upon Addition of Molecular Solvents, J. Solution Chem. 38 (2009) 589-600.

[48] A.E. Andreatta, A. Arce, E. Rodil, A. Soto, Physical properties and phase equilibria of the system isopropyl acetate + isopropanol +1 -octyl-3-methylimidazolium bis(trifluoromethylsulfonyl)imide, Fluid Phase Equilib. 287 (2010) 84-94.

[49] A.E. Andreatta, A. Arce, E. Rodil, A. Soto, Physical and excess properties of (methyl acetate + methanol + 1-octyl-3-methyl-imidazolium bis(trifluoromethylsulfonyl)imide) and its binary mixtures at $\mathrm{T}=298.15 \mathrm{~K}$ and atmospheric pressure, J. Chem. Thermodyn. 41 (2009) 1317-1323.

[50] A. Arce, E. Rodil, A. Soto, Physical and Excess Properties for Binary Mixtures of 1-Methyl-3-Octylimidazolium Tetrafluoroborate, [Omim] $\left[\mathrm{BF}_{4}\right]$, lonic Liquid with Different Alcohols, J. Solution Chem. 35 (2006) 63-78.

[51] A. Zhu, J. Wang, Q. Li, W. Jin, Volumetric and Viscosity Properties for the Binary Mixtures of 1-Octyl-3-methylimidazolium Tetrafluoroborate with Butanone or Alkyl Acetates, J. Solution Chem. 41 (2012) 2246-2256.

[52] J. Wang, A. Zhu, Y. Zhao, K. Zhuo, Excess Molar Volumes and Excess Logarithm Viscosities for Binary Mixtures of the Ionic Liquid 1-Butyl-3-methylimidazolium Hexaflurophosphate with Some Organic Compounds, J. Solution Chem. 34 (2005) 585-596.

[53] Y. Geng, S. Chen, T. Wang, D. Yu, C. Peng, H. Liu, Y. Hu, Density, viscosity and electrical conductivity of 1-butyl-3-methylimidazolium hexafluorophosphate + 
monoethanolamine and + N, N-dimethylethanolamine, J. Mol. Liq. 143 (2008) $100-108$.

[54] V. Bennett, C.W. Dikio, S.S. Angaye, D. Wankasi, E.D. Dikio, I. Bahadur, E.E. Ebenso, Interactions of 1-butyl-3-methylimidazolium hexafluorophosphate with N,N-dimethylformamide: Density and viscosity measurements, J. Mol. Liq. 219 (2016) 661-666.

[55] M.T. Zafarani-Moattar, R. Majdan-Cegincara, Viscosity, Density, Speed of Sound, and Refractive Index of Binary Mixtures of Organic Solvent + Ionic Liquid, 1Butyl-3-methylimidazolium Hexafluorophosphate at 298.15 K, J. Chem. Eng. Data. 52 (2007) 2359-2364.

[56] W. Fan, Q. Zhou, J. Sun, S. Zhang, Density, Excess Molar Volume, and Viscosity for the Methyl Methacrylate + 1-Butyl-3-methylimidazolium Hexafluorophosphate Ionic Liquid Binary System at Atmospheric Pressure, J. Chem. Eng. Data. 54 (2009) 2307-2311.

[57] P.J. Carvalho, T. Regueira, L.M.N.B.F. Santos, J. Fernandez, J.A.P. Coutinho, Effect of Water on the Viscosities and Densities of 1-Butyl-3-methylimidazolium Dicyanamide and 1-Butyl-3-methylimidazolium Tricyanomethane at Atmospheric Pressure, J. Chem. Eng. Data. 55 (2010) 645-652.

[58] N. Zec, M. Bešter-Rogač, M. Vraneš, S. Gadžurić, Volumetric and viscosimetric properties of $[$ bmim $][D C A]+y$-butyrolactone binary mixtures, J. Chem. Thermodyn. 97 (2016) 307-314.

[59] E. Quijada-Maldonado, S. van der Boogaart, J.H. Lijbers, G.W. Meindersma, A.B. 
de Haan, Experimental densities, dynamic viscosities and surface tensions of the ionic liquids series 1-ethyl-3-methylimidazolium acetate and dicyanamide and their binary and ternary mixtures with water and ethanol at $\mathrm{T}=(298.15$ to 343.15 K), J. Chem. Thermodyn. 51 (2012) 51-58.

[60] M. Geppert-Rybczyńska, J.K. Lehmann, A. Heintz, Physicochemical properties of two 1-alkyl-1-methylpyrrolidinium bis[(trifluoromethyl)sulfonyl]imide ionic liquids and of binary mixtures of 1-butyl-1-methylpyrrolidinium bis[(trifluoromethyl)sulfonyl]imide with methanol or acetonitrile, J. Chem. Thermodyn. 71 (2014) 171-181.

[61] C. Comminges, R. Barhdadi, M. Laurent, M. Troupel, Determination of Viscosity, Ionic Conductivity, and Diffusion Coefficients in Some Binary Systems: Ionic Liquids + Molecular Solvents, J. Chem. Eng. Data. 51 (2006) 680-685.

[62] M. Vraneš, A. Tot, S. Papović, N. Zec, S. Dožić, S. Gadžurić, Ideal and non-ideal behaviour of \{1-butyl-1-methylpyrrolydinium bis(trifluoromethylsulfonyl)imide + -butyrolactone\} binary mixtures, J. Chem. Thermodyn. 81 (2015) 66-76.

[63] R. Zarrougui, M. Dhahbi, D. Lemordant, Effect of Temperature and Composition on the Transport and Thermodynamic Properties of Binary Mixtures of Ionic Liquid N-Butyl-N-methylpyrrolidinium bis(Trifluoromethanesulfonyl)imide and Propylene Carbonate, J. Solution Chem. 39 (2010) 921-942.

[64] U. Domańska, M. Królikowska, Density and Viscosity of Binary Mixtures of Thiocyanate Ionic Liquids + Water as a Function of Temperature., J. Solution Chem. 41 (2012) 1422-1445. 
[65] U. Domańska, M. Laskowska, Temperature and Composition Dependence of the Density and Viscosity of Binary Mixtures of \{1-Butyl-3-methylimidazolium Thiocyanate + 1-Alcohols\}, J. Chem. Eng. Data. 54 (2009) 2113-2119.

[66] U. Domańska, M. Laskowska, Effect of Temperature and Composition on the Density and Viscosity of Binary Mixtures of Ionic Liquid with Alcohols, J. Solution Chem. 38 (2009) 779-799.

[67] U. Domańska, M. Królikowska, Density and Viscosity of Binary Mixtures of \{1Butyl-3-methylimidazolium Thiocyanate + 1-Heptanol, 1-Octanol, 1-Nonanol, or 1-Decanol\}, J. Chem. Eng. Data. 55 (2010) 2994-3004.

[68] E. Gómez, B. González, N. Calvar, Á. Domínguez, Excess molar properties of ternary system (ethanol + water +1 ,3-dimethylimidazolium methylsulphate) and its binary mixtures at several temperatures, J. Chem. Thermodyn. 40 (2008) $1208-1216$.

[69] H. Rodríguez, J. Brennecke, Temperature and composition dependence of the density and viscosity of binary mixtures of water + ionic liquid, J. Chem. Eng. Data. 51 (2006) 2145-2155.

[70] A. Haghtalab, A. Shojaeian, Volumetric and viscometric behaviour of the binary systems of $\mathrm{N}$-methyldiethanolamine and diethanolamine with 1-butyl-3methylimidazolium acetate at various temperatures, J. Chem. Thermodyn. 68 (2014) 128-137.

[71] S. Fendt, S. Padmanabhan, H.W. Blanch, J.M. Prausnitz, Viscosities of Acetate or Chloride-Based Ionic Liquids and Some of Their Mixtures with Water or Other 
Common Solvents, J. Chem. Eng. Data. 56 (2011) 31-34.

[72] C.M.S.S. Neves, P.J. Carvalho, M.G. Freire, J.A.P. Coutinho, Thermophysical properties of pure and water-saturated tetradecyltrihexylphosphonium-based ionic liquids, J. Chem. Thermodyn. 43 (2011) 948-957.

[73] U. Domańska, P. Papis, J. Szydłowski, M. Królikowska, M. Królikowski, Excess Enthalpies of Mixing, Effect of Temperature and Composition on the Density, and Viscosity and Thermodynamic Properties of Binary Systems of \{Ammonium-Based Ionic Liquid + Alkanediol\}, J. Phys. Chem. B. 118 (2014) $12692-12705$.

[74] O. Ciocirlan, O. Iulian, Properties of Pure 1-Butyl-2,3-dimethylimidazolium Tetrafluoroborate Ionic Liquid and Its Binary Mixtures with Dimethyl Sulfoxide and Acetonitrile, J. Chem. Eng. Data. 57 (2012) 3142-3148.

[75] E.J. González, L. Alonso, Á. Domínguez, Physical Properties of Binary Mixtures of the Ionic Liquid 1-Methyl-3-octylimidazolium Chloride with Methanol, Ethanol, and 1-Propanol at $T=(298.15,313.15$, and 328.15$) \mathrm{K}$ and at $P=0.1$ MPa, J. Chem. Eng. Data. 51 (2006) 1446-1452.

[76] N.I. Malek, S.P. ljardar, Binary mixtures of $\left(\left[\mathrm{C}_{4} \mathrm{mim}\right]\left[\mathrm{NTf}_{2}\right]+\right.$ molecular organic solvents): Thermophysical, acoustic and transport properties at various compositions and temperatures, J. Chem. Thermodyn. 93 (2016) 75-85.

[77] A.E. Andreatta, A. Arce, E. Rodil, A. Soto, Physico-chemical Properties of Binary and Ternary Mixtures of Ethyl Acetate + Ethanol + 1-Butyl-3-methylimidazolium bis(trifluoromethylsulfonyl)imide at $298.15 \mathrm{~K}$ and Atmospheric 
Pressure, J. Solution Chem. 39 (2010) 371-383.

[78] M.M. Akbar, T. Murugesan, Thermophysical properties of 1-hexyl-3methylimidazolium tetrafluoroborate $[\mathrm{hmim}]\left[\mathrm{BF}_{4}\right]+\mathrm{N}$-methyldiethanolamine (MDEA) at temperatures (303.15 to 323.15) K, J. Mol. Liq. 177 (2013) 54-59.

[79] A.E. Andreatta, A. Arce, E. Rodil, A. Soto, Physical Properties of Binary and Ternary Mixtures of Ethyl Acetate, Ethanol, and 1-Octyl-3-methyl-imidazolium Bis(trifluoromethylsulfonyl)imide at 298.15 K, J. Chem. Eng. Data. 54 (2009) $1022-1028$.

[80] J. Zhang, W. Wu, T. Jiang, H. Gao, Z. Liu, J. He, B. Han, Conductivities and Viscosities of the Ionic Liquid $[\mathrm{bmim}]\left[\mathrm{PF}_{6}\right]+$ Water + Ethanol and $[\mathrm{bmim}]\left[\mathrm{PF}_{6}\right]+$ Water + Acetone Ternary Mixtures, J. Chem. Eng. Data. 48 (2003) 1315-1317.

[81] N. Zec, M. Bešter-Rogač, M. Vraneš, S. Gadžurić, Physicochemical properties of (1-butyl-1-methylpyrrolydinium dicyanamide $+y$-butyrolactone) binary mixtures, J. Chem. Thermodyn. 91 (2015) 327-335.

[82] B. González, N. Calvar, E. Gómez, Á. Domínguez, Physical properties of the ternary system (ethanol + water + 1-butyl-3-methylimidazolium methylsulphate) and its binary mixtures at several temperatures, J. Chem. Thermodyn. 40 (2008) 1274-1281.

[83] J.N. Canongia Lopes, M.F. Costa Gomes, P. Husson, A.A.H. Pádua, L.P.N. Rebelo, S. Sarraute, M. Tariq, Polarity, Viscosity, and Ionic Conductivity of Liquid Mixtures Containing $\left[\mathrm{C}_{4} \mathrm{C}_{1} \mathrm{im}\right]\left[\mathrm{Ntf}_{2}\right]$ and a Molecular Component, J. Phys. Chem. B. 115 (2011) 6088-6099. 
[84] F. Kermanpour, H.Z. Niakan, Measurement and modeling the excess molar properties of binary mixtures of $\left\{\left[\mathrm{C}_{6} \mathrm{mim}\right]\left[\mathrm{BF}_{4}\right]+3\right.$-amino-1-propanol $\}$ and $\left\{\left[\mathrm{C}_{6} \mathrm{mim}\right]\left[\mathrm{BF}_{4}\right]+\right.$ isobutanol $\}$ : Application of Prigogine-Flory-Patterson theory, J. Chem. Thermodyn. 48 (2012) 129-139.

[85] F. Kermanpour, T. Sharifi, Thermodynamic study of binary mixture of $\mathrm{x}_{1}\left[\mathrm{C}_{6} \mathrm{mim}\right]\left[\mathrm{BF}_{4}\right]+\mathrm{x}_{2} 1$-propanol: Measurements and molecular modeling, Thermochim. Acta. 527 (2012) 211-218.

[86] F. Kermanpour, The excess molar properties of $\left\{\mathrm{x}_{1}\left[\mathrm{C}_{6} \mathrm{~min}\right]\left[\mathrm{BF}_{4}\right]+\mathrm{x}_{2} 2\right.$-propanol $\}$ : Application of ERAS model, J. Mol. Liq. 169 (2012) 156-162.

[87] J.L. Chevalier, P. Petrino, Y. Gaston-Bonhomme, Estimation method for the kinematic viscosity of a liquid-phase mixture, Chem. Eng. Sci. 43 (1988) 13031309.

[88] Y. Gaston-Bonhomme, P. Petrino, J.L. Chevalier, UNIFAC-VISCO group contribution method for predicting kinematic viscosity: extension and temperature dependence, Chem. Eng. Sci. 49 (1994) 1799-1806.

[89] Y. Wang, W. Hao, J. Jacquemin, P. Goodrich, M. Atilhan, M. Khraisheh, D. Rooney, J. Thompson, Enhancing Liquid-Phase Olefin-Paraffin Separations Using Novel Silver-Based Ionic Liquids, J. Chem. Eng. Data. 60 (2015) 28-36.

[90] T. Magnussen, P. Rasmussen, A. Fredenslund, UNIFAC parameter table for prediction of liquid-liquid equilibriums, Ind. Eng. Chem. Process Des. Dev. 20 (1981) 331-339.

[91] A. Fredenslund, J. Gmehling, P. Rasmussen, Vapor-liquid equilibria using 
UNIFAC : a group contribution method, Elsevier Scientific Pub. Co., 1977.

[92] D.W. Marquardt, An Algorithm for Least-Squares Estimation of Nonlinear Parameters, J. Soc. Indust. Appl. Math. 11 (1963) 431-441.

[93] H.F.D. Almeida, H. Passos, J.A. Lopes-da-Silva, A.M. Fernandes, M.G. Freire, J.A.P. Coutinho, Thermophysical Properties of Five Acetate-Based Ionic Liquids, J. Chem. Eng. Data. 57 (2012) 3005-3013.

[94] J.M. Crosthwaite, M.J. Muldoon, J.K. Dixon, J.L. Anderson, J.F. Brennecke, Phase transition and decomposition temperatures, heat capacities and viscosities of pyridinium ionic liquids, J. Chem. Thermodyn. 37 (2005) 559-568.

[95] J.M.M. Araújo, A.B. Pereiro, F. Alves, I.M. Marrucho, L.P.N. Rebelo, Nucleic acid bases in 1-alkyl-3-methylimidazolium acetate ionic liquids: A thermophysical and ionic conductivity analysis, J. Chem. Thermodyn. 57 (2013) 1-8.

[96] A. Xu, Y. Zhang, Z. Li, J. Wang, Viscosities and Conductivities of 1-Butyl-3methylimidazolium Carboxylates Ionic Liquids at Different Temperatures, J. Chem. Eng. Data. 57 (2012) 3102-3108.

[97] M.G. Freire, C.M.S.S. Neves, I.M. Marrucho, J.A.P. Coutinho, A.M. Fernandes, Hydrolysis of Tetrafluoroborate and Hexafluorophosphate Counter Ions in Imidazolium-Based Ionic Liquids, J. Phys. Chem. A. 114 (2010) 3744-3749.

[98] O. Redlich, A.T. Kister, Algebraic Representation of Thermodynamic Properties and the Classification of Solutions, Ind. Eng. Chem. 40 (1948) 345-348. 